ARTICLE

\title{
SLPI is a critical mediator that controls PTH-induced bone formation
}

\author{
Akito Morimoto ${ }^{1,2}$, Junichi Kikuta (1) 1,2,3凶 , Keizo Nishikawa²,2, Takao Sudo1,2, Maki Uenaka1,2, \\ Masayuki Furuya 1,2, Tetsuo Hasegawa 1,2, Kunihiko Hashimoto1,2, Hiroyuki Tsukazaki 1,2, Shigeto Seno (1) 4, \\ Akira Nakamura ${ }^{5}$, Daisuke Okuzaki (i] ${ }^{2,6}$, Fuminori Sugihara ${ }^{7}$, Akinori Ninomiya (1) ${ }^{7}$, Takeshi Yoshimura ${ }^{8}$, \\ Ryoko Takao-Kawabata (i) ${ }^{9}$, Hideo Matsuda (i) ${ }^{4}$ \& Masaru Ishii (i) ${ }^{1,2,3 凶}$
}

Osteoclastic bone resorption and osteoblastic bone formation/replenishment are closely coupled in bone metabolism. Anabolic parathyroid hormone (PTH), which is commonly used for treating osteoporosis, shifts the balance from osteoclastic to osteoblastic, although it is unclear how these cells are coordinately regulated by PTH. Here, we identify a serine protease inhibitor, secretory leukocyte protease inhibitor (SLPI), as a critical mediator that is involved in the PTH-mediated shift to the osteoblastic phase. S/pi is highly upregulated in osteoblasts by PTH, while genetic ablation of Slpi severely impairs PTH-induced bone formation. Slpi induction in osteoblasts enhances its differentiation, and increases osteoblast-osteoclast contact, thereby suppressing osteoclastic function. Intravital bone imaging reveals that the PTH-mediated association between osteoblasts and osteoclasts is disrupted in the absence of SLPI. Collectively, these results demonstrate that SLPI regulates the communication between osteoblasts and osteoclasts to promote PTH-induced bone anabolism.

\footnotetext{
${ }^{1}$ Department of Immunology and Cell Biology, Graduate School of Medicine and Frontier Biosciences, Osaka University, Osaka, Japan. ${ }^{2}$ WPI-Immunology Frontier Research Center, Osaka University, Osaka, Japan. ${ }^{3}$ Laboratory of Bioimaging and Drug Discovery, National Institutes of Biomedical Innovation, Health and Nutrition, Osaka, Japan. ${ }^{4}$ Department of Bioinformatic Engineering, Graduate School of Information Science and Technology, Osaka University, Osaka, Japan. ${ }^{5}$ Division of Immunology, Tohoku Medical and Pharmaceutical University, Miyagi, Japan. ${ }^{6}$ Genome Information Research Center, Research Institute for Microbial Diseases, Osaka University, Osaka, Japan. ${ }^{7}$ Core Instrumentation Facility, Immunology Frontier Research Center and Research Institute for Microbial Diseases, Osaka University, Osaka, Japan. ${ }^{8}$ Medical Affairs Department, Asahi Kasei Pharma Corporation, Tokyo, Japan. ${ }^{9}$ Laboratory for Pharmacology, Pharmaceuticals Research Center, Asahi Kasei Pharma Corporation, Tokyo, Japan. ${ }^{凶}$ email: jkikuta@icb.med.osaka-u.ac.jp; mishii@icb.med. osaka-u.ac.jp
} 
S keletal tissue undergoes dynamic remodeling throughout the life course. Bone formation by osteoblasts and bone resorption by osteoclasts occur in spatially and temporally discrete units on the bone surface, and bone resorption is followed by bone formation ${ }^{1}$. The activities of osteoblasts and osteoclasts are linked by intercellular signaling for balanced bone remodeling. Given that bone formation and resorption are tightly coupled processes in the bone microenvironment, information concerning the spatiotemporal distribution of the cells involved in these processes would provide insight into bone remodeling. Previously, we visualized osteoblasts and osteoclasts by intravital multi-photon microscopy and revealed that direct contact with osteoblasts inhibited the bone-resorbing activity of osteoclasts in vivo ${ }^{2}$. Moreover, osteoblasts and osteoclasts were segregated in a homeostatic state, whereas intermittent parathyroid hormone (PTH) treatment dynamically altered their distribution and increased their direct cell-cell interactions, thereby inhibiting bone resorption by osteoclasts.

PTH is a major endocrine inducer and stimulator of bone remodeling. PTH (1-34) (teriparatide), a biologically active Nterminal fragment of human PTH, is used clinically to increase bone mass in osteoporosis ${ }^{3,4}$. PTH stimulates osteoblasts, and indirectly activates osteoclasts by regulating receptor activator of NF- $\kappa B$ ligand (RANKL) and osteoprotegerin in osteoblast-lineage cells ${ }^{5,6}$. It has been proposed that the anabolic action of PTH requires the presence of osteoclasts. In a murine model of osteopetrosis caused by a lack of osteoclasts, the anabolic effect of PTH treatment was blunted ${ }^{7,8}$. In the clinic, concomitant treatment of osteoporosis with alendronate, an inhibitor of osteoclasts, significantly decreased the anabolic response to $\mathrm{PTH}^{9,10}$. These results imply that the shift from osteoclasts to osteoblasts contributes to the osteoanabolic effect of $\mathrm{PTH}^{11}$. However, the mechanisms that coordinately regulate these two cell types in PTH anabolism are unknown.

Secretory leukocyte protease inhibitor (SLPI) is a serine protease inhibitor, secreted in response to tissue damage that plays multiple roles in inflammation in vivo. SLPI inhibits inflammation and initiates the healing of damaged tissue ${ }^{12}$. These functions are dependent on its protease inhibition ${ }^{13,14}$ and transcriptional regulation ${ }^{15}$. Therefore, SLPI serves as a molecular switch from inflammation to tissue proliferation ${ }^{16}$.

Although expression of SLPI in osteoblasts has been reported $^{17}$, the functional role of SLPI in bone metabolism in vivo, particularly in bone resorption, has not been described. In the present study, we found that PTH strongly induced Slpi expression in osteoblasts, and the effect of PTH on bone was dependent on SLPI. Moreover, SLPI expressed by osteoblasts promoted direct cell-cell contact, thereby shifting the balance from osteoclastic activity to osteoblastic activity. Therefore, SLPI is an essential mediator that can promote a PTH-mediated switch from bone resorption to bone formation.

\section{Results}

Osteoblast Slpi expression is induced by PTH in vivo. The anabolic effect of PTH depends on its binding to the PTH receptor (PTH1R) on osteoblast-lineage cells ${ }^{11}$. Three weeks of PTH administration induced merged distribution of osteoblasts and osteoclasts (Supplementary Fig. S1a). In addition, the responses of osteoblasts and osteoclasts to PTH treatment in femoral trabecular bone were compatible to those in their calvarial counterparts (Supplementary Fig. S1b). To assess gene expression changes in response to PTH, we first isolated osteoblasts with high purity from adult bone tissues of transgenic mice, in which mature osteoblasts specifically express enhanced cyan fluorescent protein (ECFP) driven by the type I collagen promoter (i.e., Col2.3-ECFP mice) $)^{2}$. Using flow cytometry, we detected the lineage $\left(\mathrm{Lin}^{-}\right) \mathrm{CD}^{-} 5^{-} \mathrm{ECFP}^{+}$after sequential enzymatic digestion, but not from non-adherent bone marrow cells (Supplementary Fig. S1c). The ECFP ${ }^{+}$cells expressed high levels of $S p 7$ and Bglap, and so were identified as osteoblasts (Supplementary Fig. S1d).

We next evaluated transcripts in osteoblasts by RNASequencing (RNA-Seq), using a PTH mouse model ${ }^{18}$ treated intermittently with PTH or vehicle for 3 weeks. We considered genes showing a twofold or greater change to be significantly differentially expressed $(p<0.05)$; 405 genes were upregulated by PTH, while 883 were downregulated. The expression levels of the osteoblast differentiation markers $S p 7$ and Bglap were not changed (Supplementary Fig. S1e), and thus were not affected by the PTH-induced change in cell population. The mRNA level of $F g f 2$, an important growth factor for PTH anabolism ${ }^{19}$, significantly increased (Supplementary Fig. S1f, h).

In the Gene Ontology (GO) enrichment analysis, many of the upregulated GO pathways were related to the regulation of proteolysis (Supplementary Fig. S1g). Among such genes, Slpi was the highly-ranked upregulated gene induced by PTH (Fig. 1a; Supplementary Fig. S1h). Quantitative polymerase chain reaction (qPCR) confirmed that PTH upregulated the expression of Slpi in osteoblasts in vivo (Supplementary Fig. S1i). We analyzed the time-course of the effect of PTH and found that to induce an increase in the Slpi mRNA levels required 1-3 weeks of repetitive injection (Fig. 1b). PTH can have anabolic or catabolic effects, depending on its mode of administration: the anabolic effect occurs by intermittent administration of PTH, but not by continuous infusion ${ }^{20}$. Slpi upregulation was not induced by continuous infusion of PTH for 3 weeks (a catabolic regimen; Supplementary Fig. S1j).

We also analyzed Slpi expression in other cell populations in bone tissues. We examined the cell populations reported to express Slpi in an online database (Gene Expression Commons $^{21}$ ) (Supplementary Fig. S2). qPCR analyses revealed that PTH treatment increased Slpi expression in $\mathrm{Lin}^{-} \mathrm{CD}_{4} 5^{-}$ $\mathrm{CD} 31^{-} \mathrm{CD} 51^{+} \mathrm{Sca}{ }^{+}$mesenchymal stem cells (MSCs) ${ }^{22}$ (Fig. 1c), but not in total bone marrow cells, $\mathrm{CD} 45^{+} \mathrm{Ly}_{6 \mathrm{G}}{ }^{-}$ F4/80 high bone marrow monocytes/macrophages, or $\mathrm{CD} 45^{+}$ Ly6G ${ }^{+}$bone marrow granulocytes (Fig. 1c). Therefore, Slpi was specifically and highly upregulated in osteoblast-lineage cells by repetitive PTH injection in vivo.

SLPI controls the PTH-induced increase in bone mass. The above results prompted us to explore the importance of SLPI in physiological bone remodeling in vivo. Initially, we evaluated baseline bone modeling and remodeling in Slpi-knockout (KO) mice $^{23}$. In a homeostatic state, 5-week-old female mice were injected with vehicle for 6 weeks; distal femoral metaphysis was analyzed by micro-computed tomography $(\mu \mathrm{CT})$ when the mice were 11 weeks of age. Slpi-KO mice displayed no overt trabecular bone phenotype, compared with wild-type (WT) mice (Fig. 1d, f, h; Supplementary Movies 1,3 ). In vitro, WT and Slpi-KO primary osteoblasts showed identical bone-forming potential, as demonstrated by Alizarin Red S staining (Supplementary Fig. S3a). In addition, the numbers of osteoclasts differentiated from Slpi-KO macrophages were similar to the numbers differentiated from WT macrophages (Supplementary Fig. S3b). Together, these data suggest that Slpi-KO mice in a homeostatic state show normal bone remodeling with no aberrant phenotype.

To test the function of Slpi expression in PTH anabolism, we next examined the effect of PTH on Slpi-KO mice. Eleven-weekold WT mice subjected to intermittent PTH treatment for 6 weeks showed a significant increase in trabecular content, compared 
a

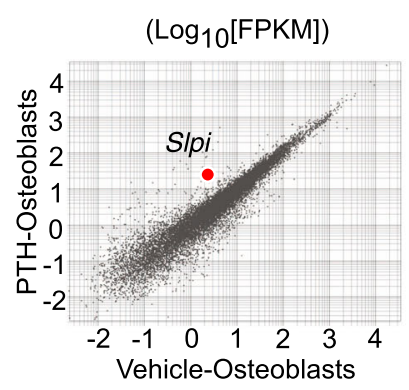

d

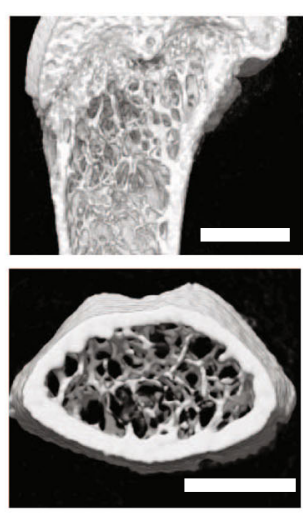

h

BV/TV

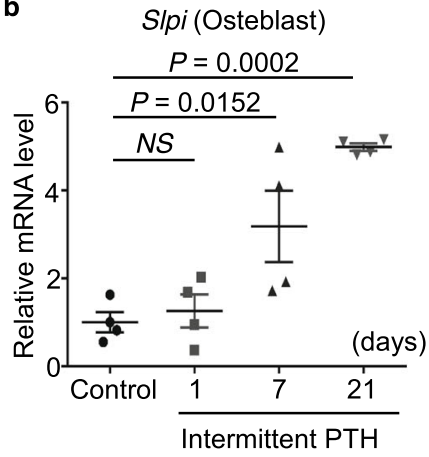

e
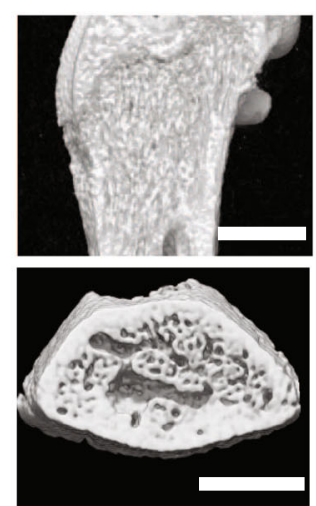

Tb.Th

interaction $P_{4}<0.0001$

$P_{6}<0.0001$

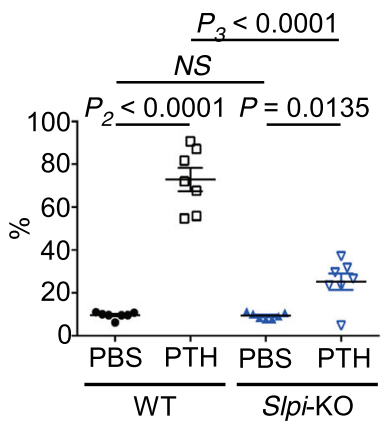

c

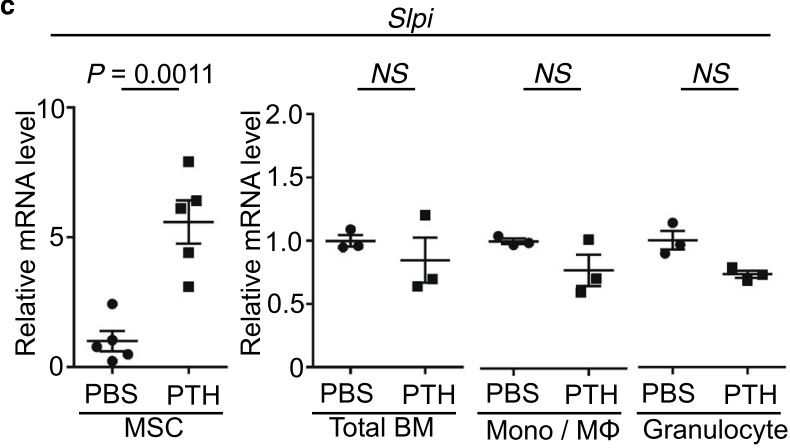

f
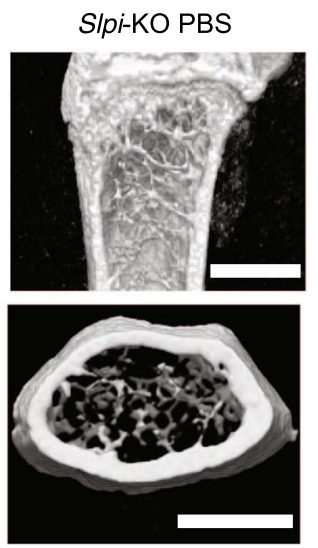

i g
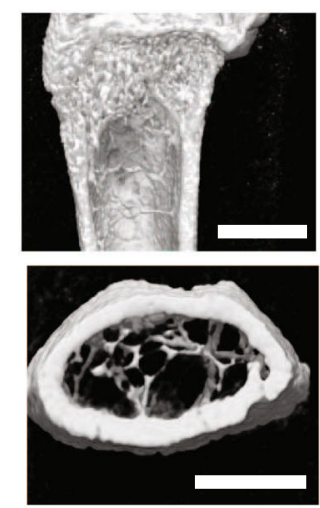

Serum CTX

interaction $P=0.0013$

interaction $P=0.0291$
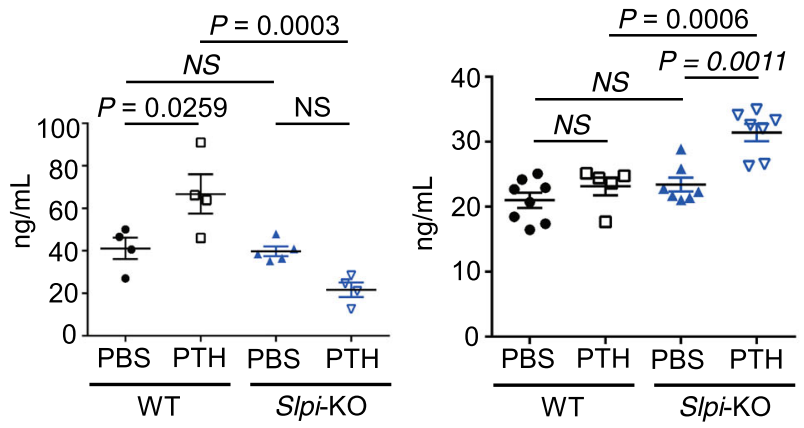

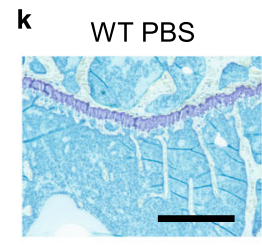

SIpi-KO PBS

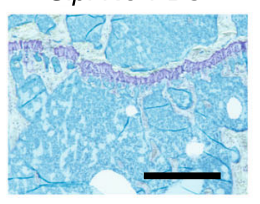

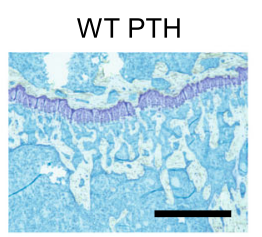

SIpi-KO PTH

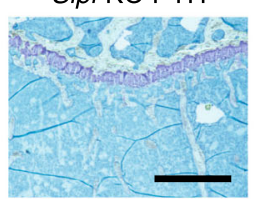

I WT PBS

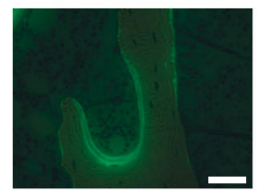

Slpi-KO PBS

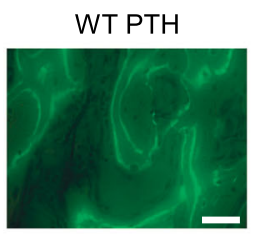

SIpi-KO PTH

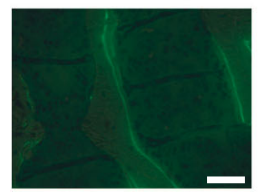

m

BFR/BS

interaction $P=0.0366$

$$
P=0.0107
$$

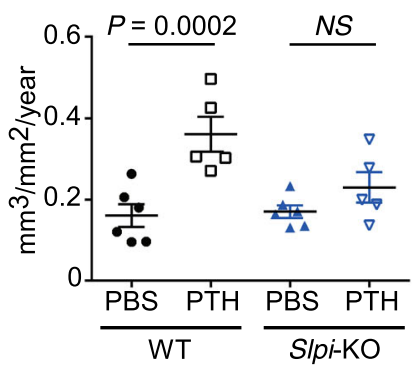

with vehicle-treated mice (Fig. 1d, e; Supplementary Movies 1, 2). This was apparent from the cancellous bone volume (BV/TV), and trabecular thickness (Tb.Th) (Fig. 1h). In contrast, the bone anabolic effect of PTH was severely disrupted in Slpi-KO mice; specifically, the rate of increase in BV/TV in Slpi-KO mice was less than half that in WT mice (Fig. 1e, g, h; Supplementary
Movies 2, 4). Similarly, PTH-treated WT mice had a significantly higher serum levels of type I procollagen N-terminal propeptide (P1NP), a biochemical marker of bone formation, compared to vehicle-treated WT control mice, which was disrupted in Slpi-KO mice (Fig. 1i). Assessment of the serum levels of C-terminal telopeptide of collagen (CTX), a biochemical marker of 


\begin{abstract}
Fig. 1 Secretory leukocyte protease inhibitor (SLPI) controls the parathyroid hormone (PTH)-induced increase in bone mass. a RNA-sequencing (RNASeq) scatter plot of differential expression between vehicle-treated osteoblasts and intermittent parathyroid hormone (PTH)-treated osteoblasts (log $10^{-}$ transformed fragments per kilobase of transcript per million mapped reads [FPKM]). $\mathbf{b}$ mRNA levels of Slpi in 7AAD- Lin- CD45- ECFP+ osteoblasts as determined by quantitative polymerase chain reaction (qPCR) analysis ( $n=4$ biologically independent samples per group). c mRNA levels of Slpi as determined by qPCR analysis ( $n=5$ mice per group for the analysis of MSC, and $n=3$ mice per group for the analysis of total BM, Mono/M $\varphi$, and granulocytes). MSC, mesenchymal stem cells (Lin ${ }^{-}$CD 45- CD31- Sca1+ CD51+ population); BM, total bone marrow cells; Mono/M $\varphi$, monocyte/ macrophage (CD45+ $\mathrm{Ly}_{6 \mathrm{G}}+$ population); granulocytes (CD45+ Ly6G- F4/80+ population). $\mathbf{d}-\mathrm{g}$ Representative micro-computed tomography images of the femurs. Scale bar, $1000 \mu \mathrm{m}$. h Ratio of bone volume to total bone volume (BV/TV), and trabecular thickness (Tb.Th) values ( $n=7$ mice per group). i Serum type I procollagen N-terminal propeptide (P1NP) levels, as measured by enzyme-linked immunosorbent assay (ELISA) (WT PBS: $n=4$ mice, WT PTH: $n=4$ mice, Slpi-KO PBS: $n=5$ mice, Slpi-KO PTH: $n=4$ mice). j Serum type I procollagen C-terminal telopeptide (CTX) levels, as measured by ELISA (WT PBS: $n=8$ mice, WT PTH: $n=5$ mice, Slpi-KO PBS: $n=7$ mice, Slpi-KO PTH: $n=7$ mice). $\mathbf{k}$ Histological analysis of the proximal tibias. Images are toluidine blue-stained sections (Representative image, $n=3$ biologically independent experiments). Scale bars, $500 \mu \mathrm{m}$. I Merged image of the calcein band and bright field of the proximal tibias (Representative image, $n=3$ biologically independent experiments). Scale bar, $50 \mu \mathrm{m}$. $\mathbf{m}$ Bone formation rate (BFR/BS) values (WT PBS: $n=6$ mice, WT PTH: $n=5$ mice, Slpi-KO PBS: $n=6$ mice, Slpi-KO PTH: $n=5$ mice). For details and statistical analysis, see Table S2. Data are means \pm SEM. NS, not significant. Statistical significance was determined by ANOVA with Dunnett's test (b), Tukey's test (h, i, j), Šidák's test $(\mathbf{m})$, and two-tailed Student's $t$-test $(\mathbf{c}) . \mathbf{h} P_{1}$ exact value $=2.8 \mathrm{E}^{-7}, P_{2}$ exact value $=8.5 \mathrm{E}^{-12}, P_{3}$ exact value $=2.8 \mathrm{E}^{-9}, P_{4}$ exact value $=5.1 \mathrm{E}^{-6}$, $P_{5}$ exact value $=2.0 \mathrm{E}^{-10}, P_{6}$ exact value $=9.5 \mathrm{E}^{-8}$.
\end{abstract}

resorption, revealed that intermittent $\mathrm{PTH}$ treatment did not increase bone resorption in WT mice, but did increase it in SlpiKO mice (Fig. 1j). Therefore, SLPI affects both bone formation and bone resorption in PTH anabolism, which results in significantly different trabecular bone phenotypes in the two genotypes. In addition, we analyzed trabecular bone in male mice to investigate the effect of sex on PTH anabolism. Five-week-old male mice were injected with vehicle or PTH for 6 weeks, and distal femoral metaphysis was analyzed by $\mu \mathrm{CT}$. The effect of PTH was weaker in WT male mice than in WT female mice, while PTH treatment decreased trabecular bone mass in Slpi-KO male mice (Supplementary Fig. S4). Therefore, these data demonstrate that the anabolic effect of PTH is also dependent on SLPI in male mice.

Given that the anabolic action of PTH results from enhanced bone formation, we examined the effect of ablation of Slpi on the cellular response to PTH via histomorphometric analysis. Twelveweek-old female WT or Slpi-KO mice were treated daily with vehicle or PTH for 4 weeks and their proximal tibias were analyzed. The effects of PTH treatment on bone formation rate (BFR/BS) were significantly reduced in Slpi-KO mice compared with WT controls (Fig. 1k-m; Supplementary Table S1). Collectively, bone histomorphometric analysis supported the notion that PTH-induced bone formation is impaired in Slpi-KO mice, consistent with the microstructural and bone metabolic marker analyses.

PTH regulates Slpi expression via PKA and Erk signaling. Next, we evaluated whether Slpi expression in osteoblasts is directly regulated by PTH in vitro. In cultures of MC3T3-E1 cells, a clonal osteoblastic cell line, PTH increased Slpi expression levels at the peak between 6 and $12 \mathrm{~h}$ after stimulation (Supplementary Fig. S5a). PTH1R is a seven G protein-coupled transmembrane protein expressed in osteoblast-lineage cells. In general, activated PTH1R selectively couples several G-protein subclasses, activating the cAMP dependent protein kinase A (PKA), intracellular $\mathrm{Ca} 2^{+}$, protein kinase $C(P K C)^{24}$, and $\beta$-arrestin-ERK1/2 25 intracellular signaling pathways. The PKA activator forskolin markedly increased Slpi expression in MC3T3-E1 cells, while the specific PKC activator phorbol 12-myristate 13-acetate (PMA), and the calcium ionophore A23187, did not upregulate Slpi (Supplementary Fig. S5b). Consistent with these findings, two pharmacological PKA inhibitors, H-89 and Rp-adenosine- $3^{\prime}, 5^{\prime}$-cyclic monophosphorothioate (Rp-cAMPS), inhibited the Slpi expression induced by PTH (Supplementary Fig. S5c). In addition, the pharmacological MEK inhibitor U0126 inhibited the PTH- induced upregulation of Slpi (Supplementary Fig. S5c). Therefore, PTH activates the cAMP/PKA and $\beta$-arrestin-ERK1/2 signaling pathways, which mediates the effect of PTH1R signaling on Slpi transcription.

Induction of Slpi expression enhances osteoblast differentiation and proliferation. To examine the biological role of SLPI in osteoblasts, we constructed a retrovirus vector for constitutive Slpi expression (pMX-Slpi-IRES-Puro), which was engineered to express both Slpi and a puromycin-resistance gene. Induction of Slpi with pMX-Slpi-IRES-Puro enhanced alkaline phosphatase (ALP) activity, and accelerated calcification in MC3T3-E1 cells and primary osteoblasts, as determined by Alizarin Red S staining (Fig. 2a). As SLPI is secreted from cells into cervical mucus or bronchial and nasal secretions ${ }^{26}$, MC3T3-E1 cells also secreted SLPI into culture medium (Fig. 2b). To examine an autocrine effect of SLPI, we added conditioned medium from Slpi-overexpressing MC3T3-E1 cells to control (mock) cells. The results showed that conditioned medium from Slpi-overexpressing cells had no effect on osteoblast differentiation (Fig. 2c). Consistently, conditioned medium from Slpi-overexpressing cells did not upregulate Runx2 (Cbfa1/AML3), the osteogenic master gene for bone formation 27,28 , the osteoblast-specific transcription factor Osterix (Sp7), or the early osteoblast marker gene Col1a1 (Supplementary Fig. S6a). The late osteoblast marker gene osteocalcin (Bglap) was slightly increased by treatment with conditioned medium (Supplementary Fig. S6a). The addition of recombinant human SLPI to the culture medium also did not enhance ALP activity in MC3T3-E1 cells (Fig. 2d). We confirmed that overexpression of Slpi upregulated Runx2, Sp7, Colla1, and Bglap in MC3T3-E1 cells (Fig. 2e; Supplementary Fig. S6b).

Next, to investigate the role of SLPI in osteoblasts, we analyzed its effects on intracellular proteins. Silver staining of intracellular proteins from MC3T3-E1 osteoblasts showed multiple enhanced bands in Slpi-overexpressing cells (Supplementary Fig. S6c). These protein extracts were analyzed by liquid chromatography tandem mass spectrometry (timsTOF pro, Bruker) and the results showed that the levels of 694 proteins were increased, while the levels of 131 proteins were decreased, by SLPI in MC3T3-E1 osteoblasts. Although SLPI is a regulator of $\mathrm{I \kappa B} \mathrm{B}^{13,29}$ and proepithelin 16 degradation, both of which activate osteoblasts $^{30,31}$, the levels of these proteins did not increase. Four proteins detected by mass spectrometry analysis were associated with "positive regulation of osteoblast differentiation," according to NCBI GO annotations. Of those proteins, catenin beta-1 ( $\beta$-catenin) was upregulated by SLPI, and higher levels of 

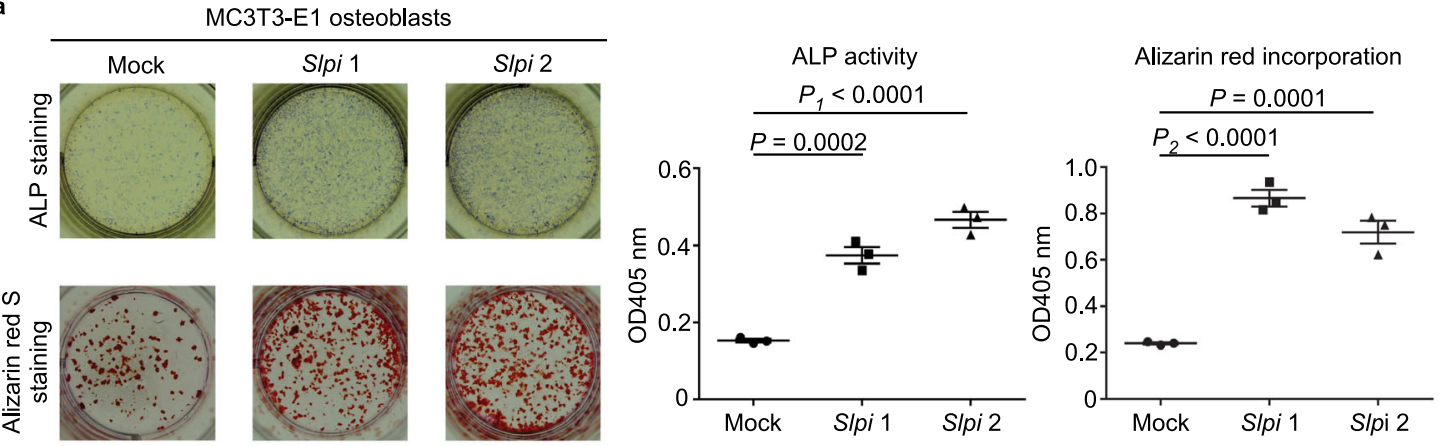
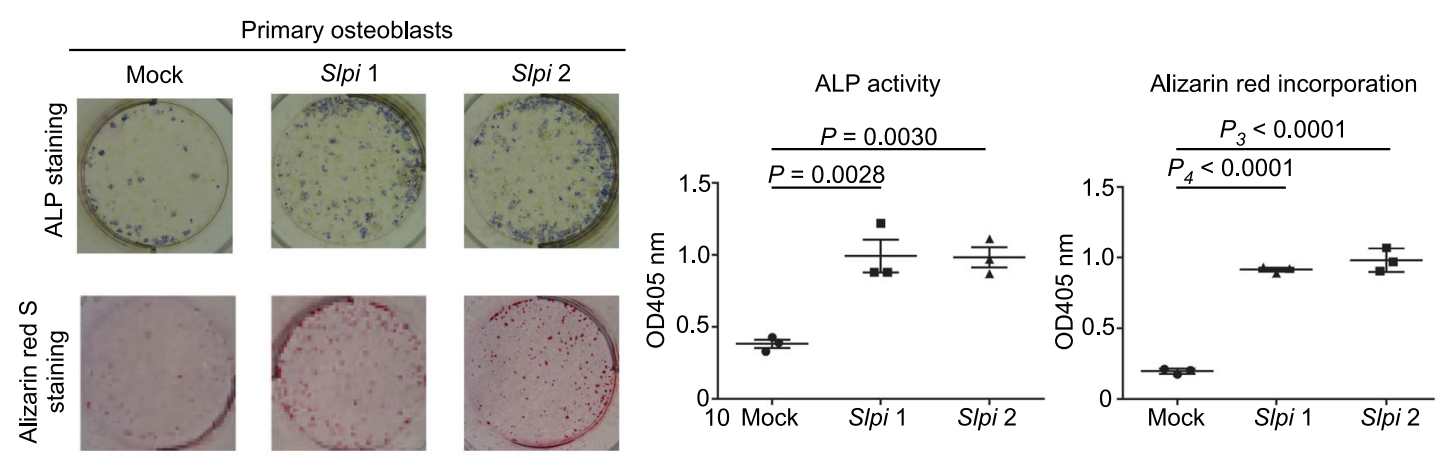

b c

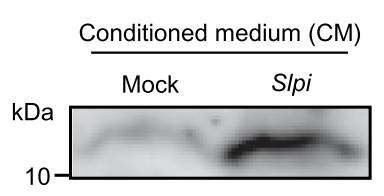

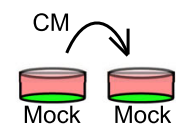
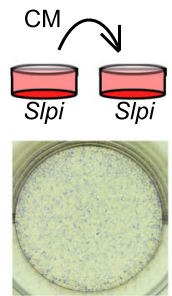

Col1a1

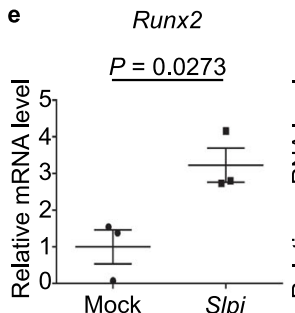

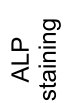

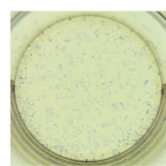

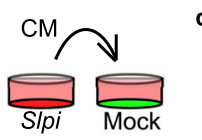

d

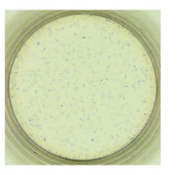

Vehicle

Recombinant human SLPI

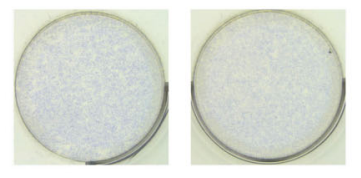

f

Proliferation

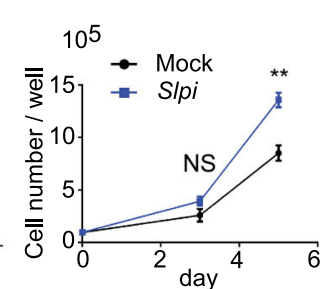

Fig. 2 Induction of SIpi enhances osteoblast differentiation and proliferation. a Representative images of MC3T3-E1 and primary mouse calvarial osteoblasts transfected with either pMX-SIpi-IRES-puro or pMX-IRES-puro by alkaline phosphatase (ALP) staining (upper) and Alizarin Red S staining (lower) ( $n=3$ biological replicates per group). $\beta$-glycerophosphate was used at a concentration of $10 \mathrm{mM}$. $\mathbf{b}$, Immunoblot of SLPI in conditioned medium from control MC3T3-E1 and Slpi-overexpressing cells (Representative blot, $n=2$ biologically independent experiments). c Typical images of ALP staining of MC3T3-E1 cells. Control (mock) or Slpi overexpressing (SIpi) MC3T3-E1 cells were treated with mock or Slpi conditioned medium under osteogenic conditions for 3 days. d ALP-stained images of MC3T3-E1 cells treated with vehicle or human recombinant SLPI (10 $\mu \mathrm{g} / \mathrm{mL})$ under osteogenic conditions for 3 days. e qPCR analysis of MC3T3-E1 cells transfected with pMX-S/pi-IRES-puro or pMX-IRES-puro ( $n=3$ biological replicates per group). Cells were cultured in osteogenic differentiation medium for 3 days, and the expression levels of osteoblast transcript regulators (Runx2, Sp7) and differentiation marker genes (Bglap, Col1a1) were quantified. f Proliferation of MC3T3-E1 cells transfected with pMX-Slpi-IRES-puro or pMX-IRES-puro. Cells were cultured in $\alpha$ - MEM for 72 or $120 \mathrm{~h}$ after adherence ( $n=3$ biological replicates per group). ${ }^{\star} P=0.0071$. Data are means \pm SEM. NS, not significant. Statistical significance was determined by ANOVA with Dunnett's test (a), and two-tailed Student's $t$-test $(\mathbf{e}, \mathbf{f})$. a $P_{1}$ approximate value $<\mathrm{E}^{-15}, P_{2}$ approximate value $<\mathrm{E}^{-15}, \mathrm{P}_{3}$ approximate value $<\mathrm{E}^{-15}$, and $\mathrm{P}_{4}$ approximate value $<\mathrm{E}^{-15}$.

$\beta$-catenin protein were detected by immunoblotting in Slpioverexpressing cells (Supplementary Fig. S6d). Consistent with regulation of intracellular $\beta$-catenin levels by the proteasomal degradation pathway, the mRNA levels of $\beta$-catenin were not increased by SLPI (Supplementary Fig. S6e). Therefore, canonical
Wnt signaling is a candidate downstream target of SLPI, although the mechanistic relationship between SLPI and the ubiquitinproteasome pathway is unclear.

Because osteoblast proliferation is also an important indicator of osteogenesis, we analyzed the proliferation of cultured MC3T3- 
E1 cells. Compared with mock MC3T3-E1 cells, the numbers of Slpi-overexpressing cells began to increase at day 3; the difference was statistically significant at day 5 (Fig. 2f), indicating that SLPI promotes osteoblast proliferation. The anabolic response of osteoblasts to PTH is in part due to reduced apoptosis of osteoblasts and their precursors ${ }^{32}$. Therefore, we evaluated the antiapoptotic effect of SLPI on MC3T3-E1 cells by flow cytometry. Neither $7 \mathrm{AAD}^{-}$annexin $\mathrm{V}^{+}$(early apoptotic) cell counts nor $7 \mathrm{AAD}^{+}$annexin $\mathrm{V}^{+}$(late apoptotic) cell counts were increased by Slpi overexpression (Supplementary Fig. S6f).

The above findings show that SLPI promotes osteoblast proliferation and differentiation by targeting key transcription factors such as $\beta$-catenin, Runx2, and Osterix.

SLPI is associated with adhesion between osteoblasts and osteoclasts. To determine how SLPI affects bone resorption, we explored the paracrine role of SLPI in signaling between osteoblasts and osteoclasts. Because SLPI is a regulator of nuclear factor kappa B (NF-kB) in monocyte-lineage cells, we examined whether osteoclast differentiation via NF- $\kappa B$ signaling ${ }^{33}$ was modulated by SLPI secreted from osteoblast-lineage cells. Treatment with recombinant human SLPI did not affect RANKLinduced osteoclast differentiation in vitro (Fig. 3a). Osteoclastic bone degradation is mediated by cysteine proteases and matrix metalloproteinases, which are not considered mechanistic targets of the serine proteinase inhibitor SLPI ${ }^{34-36}$. Consistent with this, we found that osteoclast pit-forming activity in vitro was not affected by recombinant human SLPI (Fig. 3b). Therefore, SLPI secreted from osteoblasts does not directly affect osteoclast differentiation or bone-resorbing activity.

Proteases and protease inhibitors are associated with cellular detachment from surrounding components. Indeed, SLPI in osteoblasts is reportedly associated with focal cell adhesion ${ }^{37}$. Therefore, we examined the effect of SLPI on adhesion between osteoblasts and osteoclasts by coculture experiments. When control (mock) MC3T3-E1 cells were cultured with primary osteoclasts, the duration of osteoblast-osteoclast colocalization was generally less than $1 \mathrm{~h}$ (Fig. 3c, d; Supplementary Movie 5). In contrast, induction of Slpi in MC3T3-E1 cells slightly increased the ratio of long cell-cell contact (Fig. 3c, d; Supplementary Movie 6). Consistent with these findings, quantitative analysis revealed that contact duration was significantly increased by Slpi induction (Fig. 3e). Also, Slpi mRNA was expressed in EGFP + osteoblastic cells, but not in tdTomato ${ }^{+}$osteoclasts, in coculture experiments (Fig. 3f).

Given that SLPI promotes leukocyte chemotaxis in vitro ${ }^{38}$, we examined whether SLPI secreted from osteoblasts directly attracts osteoclasts. We evaluated the effects of SLPI inhibition by commercially available neutralizing antibodies on osteoclast mobility. Time-lapse imaging was performed to assess cocultures of MC3T3-E1 cells and primary osteoclasts until $12 \mathrm{~h}$ after treatment with an SLPI neutralizing antibody or an IgG isotypematched control antibody. However, treatment with an SLPI neutralizing antibody did not inhibit the osteoblast-osteoclast contact enhanced by SLPI (Supplementary Fig. S7).

Next, to investigate the molecular mechanisms by which Slpi induces colocalization of osteoblasts with osteoclasts further, we performed an RNA-Seq analysis using control (mock) MC3T3-E1 cells and Slpi-overexpressing cells (Supplementary Fig. S8a). Chemokines and growth factors have key roles in physiological bone remodeling by controlling migration, and localization of osteoclasts and their precursors 39,40 . However, these factors were overall not remarkably changed by SLPI in osteoblasts (Supplementary Fig. S8a). Similarly, we did not observe reduced expression of the repulsive guidance factor Plexin-B1, which is known to contribute to the spatial segregation of osteoblasts and osteoclasts $^{41,42}$ (Supplementary Fig. S8a).

Notably, among the canonical pathways affected by SLPI, Wnt/ $\beta$-catenin signaling was one of the most significantly affected (Supplementary Fig. S8b), consistent with the regulation of $\beta$-catenin by SLPI (Supplementary Fig. 6d). RhoA signaling was another pathway significantly affected by SLPI (Supplementary Fig. S8b). Rho-associated coiled-coil kinases (ROCKs) are pivotal downstream effectors of RhoA. Immunoblot analysis revealed that ROCK2, but not ROCK1, was upregulated at the protein level in Slpi-overexpressing cells (Supplementary Fig. S8c). ROCK inhibitor Y-27632 significantly prevented the osteoclast-osteoblast contact that had been promoted by SLPI, suggesting that ROCK is associated with Slpi-induced osteoblastosteoclast adhesion (Supplementary Fig. S8d). Moreover, Slpioverexpressing MC3T3-E1 cells had comparably enhanced transmembrane glycoprotein ICAM-1 expression (Supplementary Fig. S8e). These results were consistent with previous studies showing that RhoA signaling is associated with heterotypic cell-cell adhesion, such as hematopoietic stem cell-niche cell adhesion $^{43}$, and osteoblast-osteoclast adhesion ${ }^{44}$, through the expression of transmembrane cell adhesion molecules ${ }^{44,45}$.

Next, we analyzed how interactions between osteoblasts and osteoclasts influenced the activities of both cell types. With respect to bone formation, coculture of osteoblasts and osteoclasts reportedly promoted mineralization in osteoblasts ${ }^{46}$. Consistent with this observation, the presence of differentiated osteoclasts led to markedly increased ALP activity in primary osteoblasts (Supplementary Fig. S9a, b). Alizarin Red S staining revealed that the presence of mature osteoclasts led to markedly increased mineralization in primary osteoblasts (Supplementary Fig. S9a, b). Notably, direct coculture with mature osteoclasts for $24 \mathrm{~h}$ significantly increased Slpi expression in MC3T3-E1 cells (juxtacrine interaction in Fig. 3g). In addition, the expression of Fgf2, which was upregulated in PTH-treated osteoblasts, was increased in the presence of osteoclasts. We also performed experiments using the Transwell system, which enables sharing of culture medium between osteoblasts and osteoclasts without direct contact. However, Slpi and Fgf2 expression in osteoblasts did not increase under this condition (paracrine interaction in Fig. 3g), suggesting that direct osteoblast-osteoclast contact plays a role in increasing the levels of these anabolic factors. With respect to bone resorption, the presence of differentiated MC3T3-E1 cells inhibited bone marrow macrophages from undergoing osteoclastogenesis (Supplementary Fig. S9c). Resorption pit formation by mature osteoclasts was also reduced during coculture with differentiated MC3T3-E1 cells (Supplementary Fig. S9d). Collectively, these results suggest that the close contact between osteoblasts and osteoclasts would result in increased bone formation and decreased bone resorption. Therefore, SLPI-induced colocalization presumably promotes interactions between osteoblasts and osteoclasts, modifying the activities of both cell types to facilitate the anabolic action of PTH.

The PTH-induced direct association between osteoblasts and osteoclasts was disrupted in the absence of Slpi. Cell-to-cell attraction and cell stickiness promote the intermingling of different cell types, whereas tension between cells results in their segregation ${ }^{47}$. Therefore, we used intravital multi-photon microscopy to determine whether SLPI-mediated heterophilic interaction impacted PTH-induced bone cell distribution in vivo. We developed a system enabling simultaneous visualization of osteoblasts and osteoclasts using double transgenic Col2.3-ECFP/tartrate-resistant acid phosphatase (TRAP)tdTomato reporter mice ${ }^{2}$. We crossed these mice with Slpi-KO mice, and compared the living bone tissue of anabolic PTH-treated mice. 


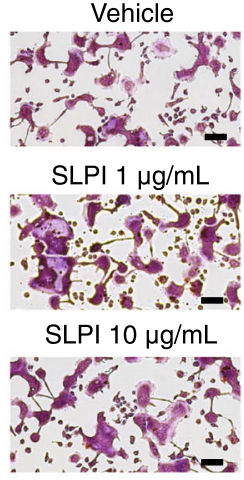

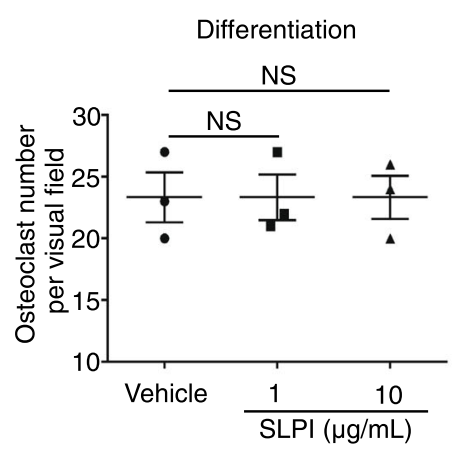

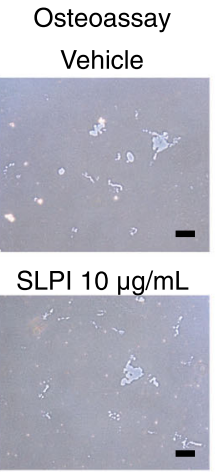

Resorption activity

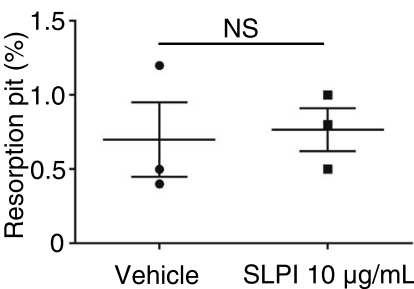

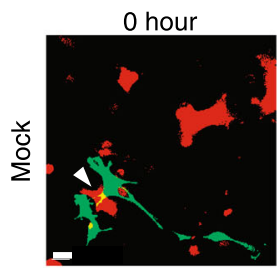
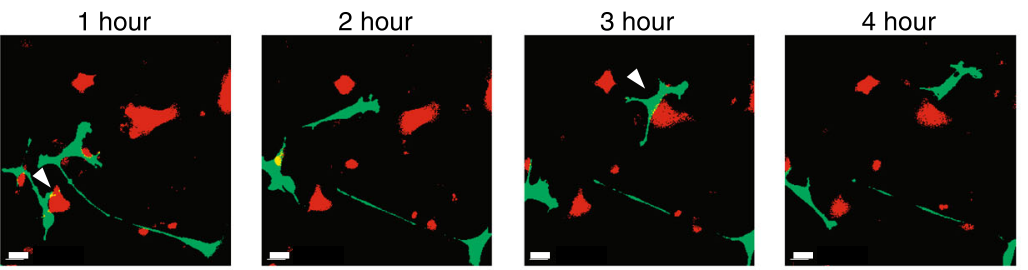

$\overline{\overline{0}}$
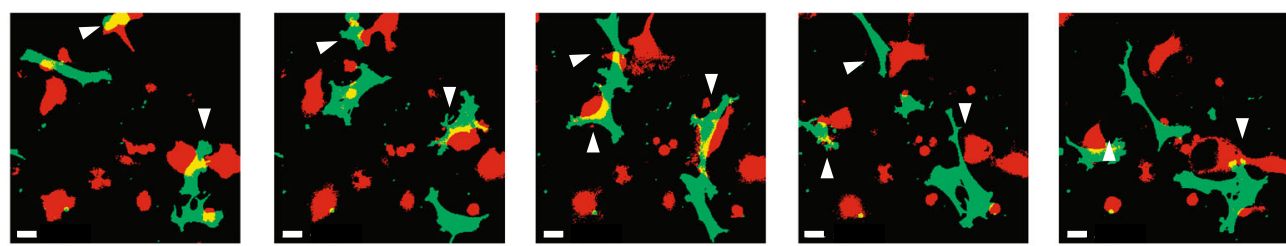

MC3T3-E1-EGFP TRAP-tdTomato+ primary osteoclasts - Cell-cell contact

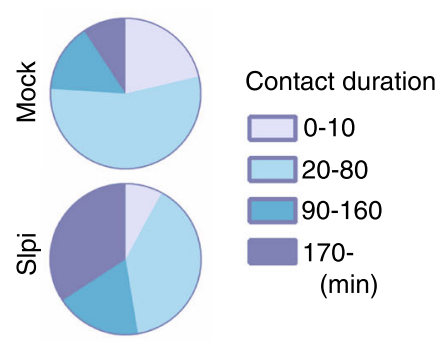

e

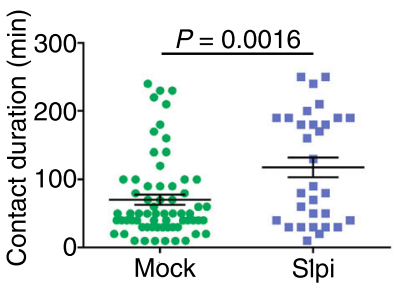

f

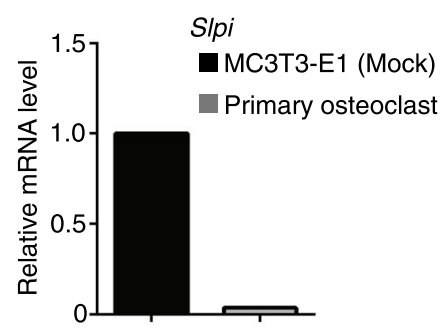

g
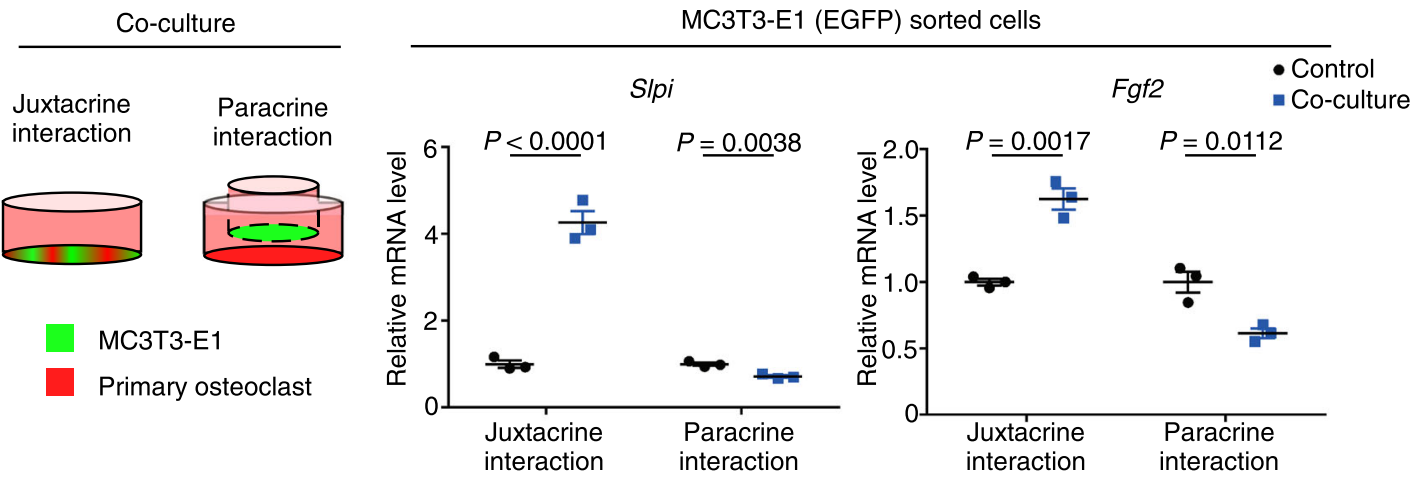

$\mathrm{ECFP}^{+}$osteoblasts and tdTomato ${ }^{+}$osteoclasts were increased after 3 weeks of PTH treatment (Fig. $4 \mathrm{a}-\mathrm{c}$ ). The densities of $\mathrm{ECFP}^{+}$ osteoblasts and tdTomato ${ }^{+}$osteoclasts between Slpi-KO and WT mice were similar after PTH treatment (Fig. $4 \mathrm{a}-\mathrm{c}$ ). However, the degrees of osteoblast and osteoclast gathering both increased, and their intermingling was disrupted by knockout of Slpi. Quantification of the cell mixture index (CMI), a cell mixture parameter calculated mathematically by hierarchical clustering ${ }^{2}$, demonstrated that the intermingling enhanced by PTH was partially but significantly disrupted in Slpi-KO mice, compared to WT mice (Fig. 4a, d; Supplementary Fig. S10). Consistent with this, the number of dynamic osteoblast-osteoclast contact events normalized to the surface area of osteoblasts or osteoclasts was significantly decreased in Slpi-KO mice, according to three-dimensional co-localization analysis of 
Fig. 3 SLPI is associated with adhesion between osteoblasts and osteoclasts. a TRAP-stained cells showing the effect of recombinant human SLPI on osteoclastogenesis. Scale bar, $100 \mu \mathrm{m}$. Right, numbers of TRAP ${ }^{+}$cells $(n=3$ biological replicates per group). $\mathbf{b}$ Effects of SLPI on the generation of bone resorption pits. Mature osteoclasts were transferred to a Corning Osteoassay Surface in the presence or absence of recombinant human SLPI. After $48 \mathrm{~h}$, cells were removed, and resorption pits were visualized. Scale bar, $200 \mu \mathrm{m}$. Right, area of resorption pits (\%) ( $n=3$ biological replicates per group). c Fluorescence images of mock (upper panel) or Slpi-overexpressing (lower panel) MC3T3-E1-EGFP cells in contact with TRAP-tdTomato+ primary osteoclasts (Representative image, $n=3$ biologically independent experiments). Confocal fluorescence microscopy images were acquired at $0,120,240$, 360 , and $480 \mathrm{~min}$. White triangles, direct cell-cell contact. Scale bar, $10 \mu \mathrm{m}$. d, e Analysis of osteoblast-osteoclast contact duration based on the data in c (Mock: $n=67$ cells examined over six independent experiments, Slpi: $n=31$ cells examined over 5 independent experiments). $\mathbf{f}$ Relative expression levels of Slpi in enhanced green fluorescent protein (EGFP)-labeled MC3T3-E1 cells and tdTomato-labeled osteoclasts. After coculture, MC3T3-E1 cells and osteoclasts were separated ( $n=1$ experiment per group). $\mathbf{g}$ Left, diagrammatic representation of in vitro coculture of osteoclasts with MC3T3-E1-EGFP cells. Right panel shows the effects of coculture on Slpi and Fgf2 expression in MC3T3-E1-EGFP cells ( $n=3$ biological replicates per group). Data are means \pm SEM. NS, not significant. Statistical significance was determined by ANOVA with Dunnett's test (a), and two-tailed Student's $t$-test (b, e, $\mathbf{g}$ ).

time-lapse images (Fig. 4e-g). These results demonstrate that SLPI changes bone cell distribution, resulting in altered cell-cell communication in the setting of PTH anabolism in vivo.

\section{Discussion}

Bone remodeling is achieved by the orchestration of two cell types that have opposite functions, i.e., bone-resorbing osteoclasts and bone-forming osteoblasts. PTH has been used clinically to stimulate bone formation by enhancing the activities of osteoblasts and osteoclasts in a coordinated manner, although the precise underlying mechanisms remain unclear ${ }^{11,48}$. In this study, we showed that Slpi is one of the genes most highly upregulated by PTH in osteoblasts and is critical for the osteoanabolic action of PTH. SLPI exerts two distinct functions: (1) it acts within osteoblasts themselves and enhances bone formation by controlling gene expression, and (2) it enhances adhesive force to neighboring osteoclasts, thereby increasing cell-cell communication between osteoblasts and osteoclasts $^{2}$ (Fig. 5). Therefore, SLPI expression by osteoblasts concurrently regulates the activity of osteoblasts themselves, and their surrounding microenvironment.

Similar bifunctional activity has also been reported for some molecules related to innate immunity and tissue regeneration, such as high mobility group box-1 (HMGB1) and thymidine phosphorylase (TYMP). HMGB1 is a nuclear DNA-binding protein that is involved in transcriptional regulation by $\mathrm{p} 53$ or NF- $\kappa$ B. On the other hand, upon its release from dead or damaged cells, HMGB1 also acts as a ligand that activates macrophages and dendritic cells through damage-associated molecular patterns ${ }^{49}$. TYMP, also known as platelet-derived endothelial cell growth factor, catalyzes the hydrolysis of thymidine to ensure the availability of a pyrimidine nucleotide pool ${ }^{50}$. TYMP is secreted and promotes angiogenesis by inducing proliferation and migration of endothelial cells ${ }^{51,52}$. While the functions seem to be independent in these examples, the function of SLPI is characteristic because both functions contribute to the same consequence: an increase in bone mass. SLPI is a reasonable candidate mediator for finely controlling bone metabolism.

In addition to the interaction between osteoblasts and mature osteoclasts, the recruitment of osteoclast precursors (or monocytes/macrophages) might support the anabolic activity of PTH. Indeed, some types of macrophages are associated with PTH anabolism in bone ${ }^{53}$. Previous studies have shown that the migration of osteoclast precursors is regulated by chemokines (e.g., CCR1 chemokines ${ }^{39}$ ), and growth factors (e.g., platelet derived growth factor $\mathrm{BB}^{40}$ ). In our model, the expression levels of Ccl5 and Pdgfa were increased in Slpi-overexpressing osteoblasts, implying that these chemotactic factors may contribute to the attraction of osteoclast precursors (Supplementary Fig. S7a). Moreover, given the partial contribution of SLPI to PTH-induced change in CMI in the present study, there might be an additional, $S l p i$-independent pathway for regulation of bone cell re- distribution. Particularly, cysteine-X-cysteine family chemokine ligand $1(\mathrm{Cxcl})$ is highly upregulated in osteoblasts by PTH treatment, and direct osteoclast chemotactic migration ${ }^{54}$. Although the analytical method to evaluate osteoblast-osteoclast precursor communication has not been established yet, the roles of such chemokines in PTH anabolism, as well as migration of osteoclast precursors, should be investigated in the future.

In general, matrix metalloproteases have been considered to play roles in cellular detachment from surrounding components ${ }^{55}$, while serine proteases may also be involved in cell adhesion ${ }^{56,57}$. SLPI plays a role in focal adhesion of cancer cells to the surrounding environment ${ }^{58}$. In our analysis, we focused on the fact that RhoA signaling and ROCK2 protein were affected by the expression of SLPI, as RhoA signaling is associated with cell-cell adhesion through the expression of transmembrane cell adhesion molecules. Given such multiple roles for RhoA signaling, further studies are needed to determine the in vivo role of ROCK proteins in the anabolic state of PTH.

Finally, we should also consider the limitations of this study. Bone histomorphometry showed that intermittent PTH treatment did not significantly change the osteoclast parameters, osteoclast surface/bone surface (Oc.S/BS) or eroded surface (ES/BS), in either WT or Slpi-KO mice (Supplementary Table S1). This was partially because bone histomorphometry works well for evaluation of bone formation, but it is less reliable when used to evaluate the dynamism of bone resorption ${ }^{59}$. Oc.S/BS and ES/BS are static measures of resorption surfaces, but provide no direct information concerning the dynamic status of osteoclast activity. In particular, bone histomorphometry did not necessarily reflect PTHactivated bone resorption both in our data and in previous reports by another group ${ }^{60}$. To evaluate the dynamic feature of osteoclast activity influenced by PTH, we analyzed serum CTX levels ${ }^{61}$, and showed that intermittent PTH treatment did not increase bone resorption in WT mice, but did increased it in Slpi-KO mice, suggesting a substantial effect of SLPI on bone resorption in $\mathrm{PTH}$ anabolism.

In conclusion, based on in vivo screening of gene expression in osteoblasts, we analyzed the bone phenotype of Slpi-KO mice and found that SLPI was strongly associated with PTH anabolism. Analysis of the molecular and cellular networks related to PTH anabolism will provide effective information in terms of the optimal usage of this drug and may facilitate the development of therapies for intractable bone diseases.

\section{Methods}

Mice. C57BL/6J mice were obtained from CLEA Japan (Tokyo, Japan). Slpi-KO mice were kindly provided by A. Nakamura's laboratory (Tohoku Medical and Pharmaceutical University, Sendai, Japan $)^{23}$. The generation of Col2.3-ECFP and TRAP-tdTomato mice was described previously 2,62 . Mice were randomized to the treatment or control group. The mice were bred and maintained under specific pathogen-free conditions at the animal facilities of Osaka University (Osaka, 


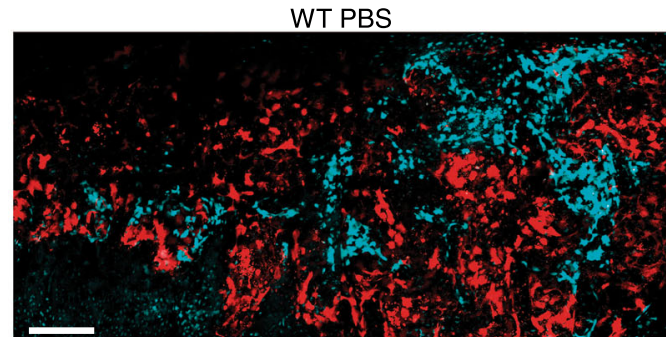

SIpi-KO PBS

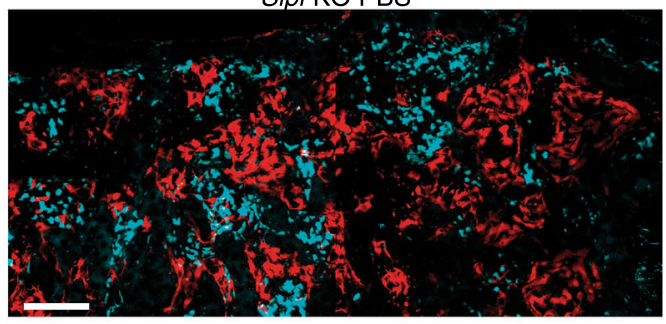

WT PTH

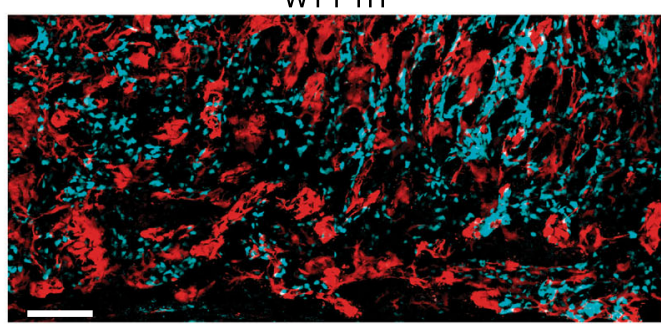

SIpi-KO PTH

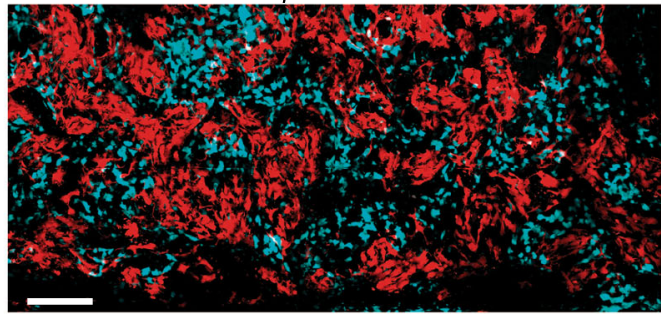

b

Osteoblast area

Interaction $P=0.0345$

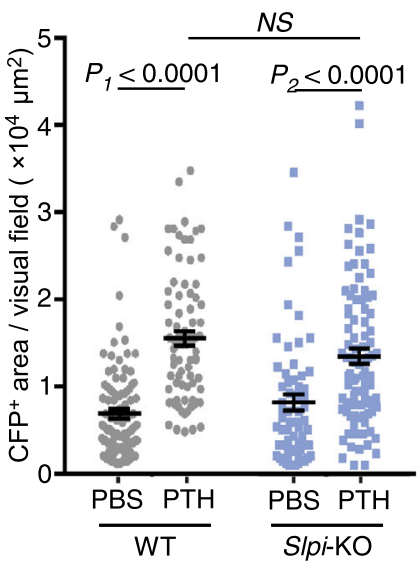

e

WT PBS
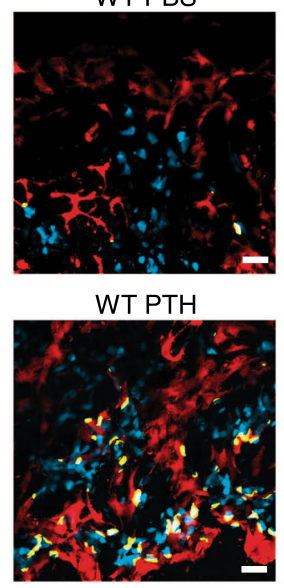

C

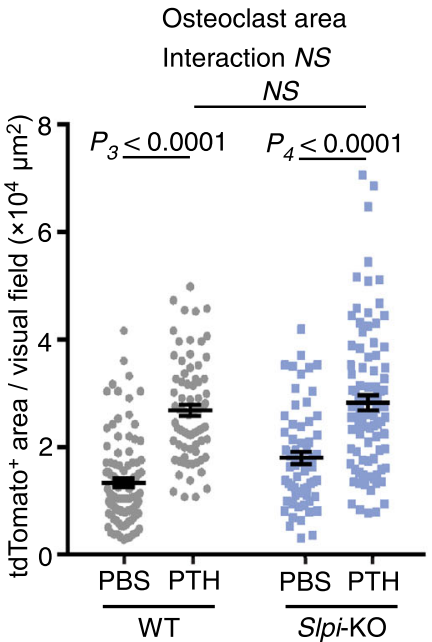

f Contact N. / OB surface area Interaction $P=0.0001$ $P_{7}<0.0001$

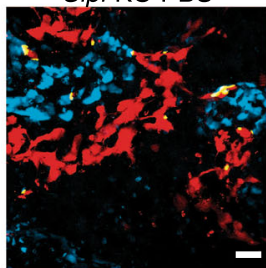

SIpi-KO PTH

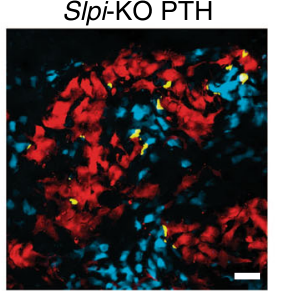

$001 P=0.0055$

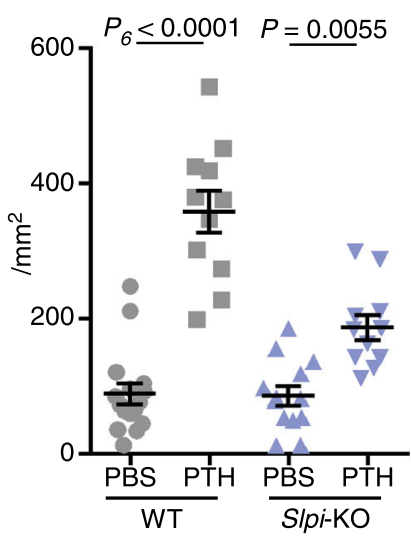

d

Distribution

Interaction $P=0.0347$

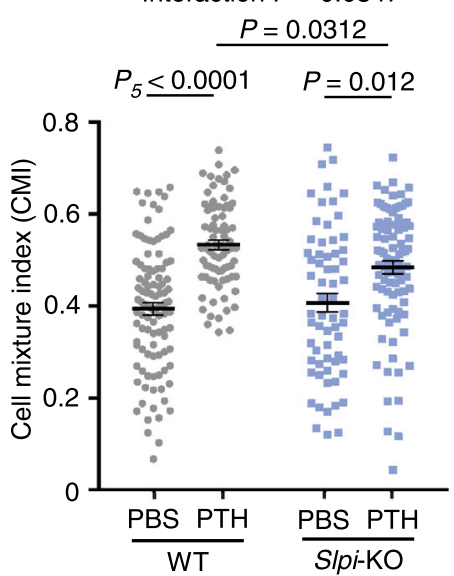

g Contact N. / OC surface area Interaction $P=0.0193$

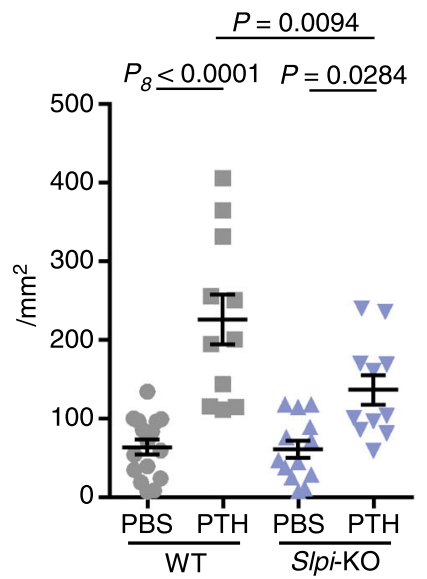

Japan). These mice were maintained in 12 -h light/12-h dark cycles, and the housing temperature and humidity were $23 \pm 1.5^{\circ} \mathrm{C}$ and $45 \pm 15 \%$, respectively. We have complied with all relevant ethical regulations for animal testing and research. Animal experiments were performed in accordance with the experimental animal guidelines of Osaka University under approved protocols.
PTH administration. Intermittent PTH injections were administered to the mice (vehicle, phosphate-buffered saline [PBS], or human PTH (1-34) [teriparatide; Asahi Kasei Pharma Corporation, Tokyo, Japan]). The dose of hPTH (1-34) was $40 \mu \mathrm{g} / \mathrm{kg}$ body weight per day, or vehicle on 5 days per week, delivered via subcutaneous injection for the indicated period, as described previously ${ }^{18}$. 
Fig. 4 The PTH-induced direct association between osteoblasts and osteoclasts was disrupted in the absence of SLPI. a Maximum-intensity projection (MIP) images of skull tissues obtained by intravital microscopy (Representative image, $n=6$ biologically independent experiments). Left panel, tiling image of Col2.3-ECFP/TRAP-tdTomato/S/piWT/WT mice; right panel, image of Col2.3-ECFP/TRAP-tdTomato/S/piKO/KO mice at 3 weeks after intermittent PBS (upper panel) or PTH (lower panel) treatment. Cyan, mature osteoblasts; red, mature osteoclasts. Scale bar, $200 \mu \mathrm{m}$. b Areas of ECFP+ mature osteoblasts per visual field in the tiling image. Cyan ${ }^{+}$(mature osteoblast) areas were binarized using Otsu's thresholding method. c Areas of tdTomato ${ }^{+}$mature osteoclasts per visual field in the tiling image. tdTomato+ (mature osteoclast) areas were binarized using Otsu's thresholding method. $\mathbf{d}$ Cell mixture index (CMI) values per visual field in WT mice. b-d WT PBS: $n=103$ images from six mice, WT PTH: 80 images from five mice, Slpi-KO PBS: 64 images from five mice, SIpi-KO PTH: 93 images from six mice. e, MIP images of 3D colocalization. Left panel, Col2.3-ECFP/TRAP-tdTomato/SlpiWT/WT mice; right panel, Col2.3-ECFP/TRAP-tdTomato/SIpiKO/KO mice, at 3 weeks after intermittent PBS (upper panel) or PTH (lower panel) treatment (Representative image, $n=6$ biologically independent experiments). Cyan, mature osteoblasts; red, mature osteoclasts. Contact areas (yellow) were defined as the areas of osteoblast and osteoclast colocalization. Scale bar, $200 \mu \mathrm{m}$.Numbers of contacts between osteoblasts and osteoclasts; data were normalized to the surface area of ECFP ${ }^{+}$osteoblasts (f), and tdTomato ${ }^{+}$osteoclasts (g), respectively (WT PBS: $n=11$ mice, WT PTH: 11 mice, Slpi-KO PBS: 16 mice, Slpi-KO PTH: 13 mice). Data are means \pm SEM. NS, not significant. Statistical significance was determined by ANOVA with Tukey's test (b, $\mathbf{c}, \mathbf{f}, \mathbf{g})$, and by Welch's ANOVA followed by Bonferroni's multiple comparisons test $(\mathbf{d}) . \mathbf{b} P_{7}$ exact value $=3.4 \mathrm{E}^{-13}, P_{2}$ exact value $=9.6 \mathrm{E}^{-9}$. $\mathbf{c} P_{3}$ exact value $=4.3 \mathrm{E}^{-13}, P_{4}$ exact value $=$ $4.9 \mathrm{E}^{-5}$. d $P_{5}$ approximate value $<\mathrm{E}^{-15}$. f $P_{6}$ exact value $=2.1 \mathrm{E}^{-12}, P_{7}$ exact value $=4.2 \mathrm{E}^{-6} . \mathbf{g} P_{8}$ exact value $=2.5 \mathrm{E}^{-7}$.
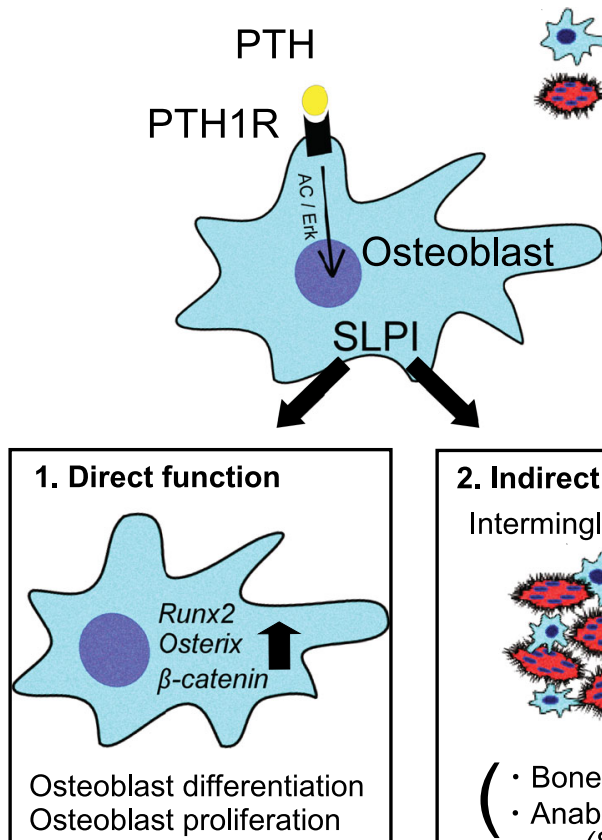

2. Indirect function Intermingling distribution

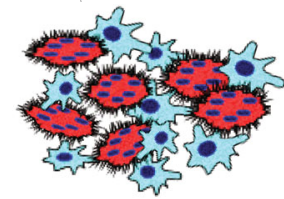

(- Bone resorption 1 (Slpi, Fgf2)

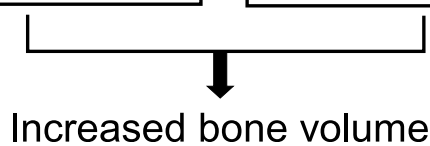

Fig. 5 Model of SLPI functions in bone metabolism. Parathyroid hormone (PTH) binding to parathyroid hormone 1 receptor (PTH1R) upregulates SLPI expression via the pathways of adenylyl cyclase $(A C)$ /protein kinase $A$, and Erk. SLPI directly acts in osteoblasts to enhance bone formation by controlling gene expression. Additionally, SLPI promotes the adhesion of osteoblasts to neighboring osteoclasts, thereby increasing direct cell-cell contact. This indirect effect creates a microenvironment in the reversal phase, leading to stimulation of Slpi and Fgf2 expression in osteoblasts, and inhibition of osteoclastic bone resorption.

For RNA-seq and qPCR analysis, 12-week-old female Col2.3-ECFP mice were treated with PTH and evaluated at 1 day, 1 week, and 3 weeks later. Osteoblasts were harvested at $24 \mathrm{~h}$ after the final injection of intermittent PTH

For continuous PTH administration, osmotic pumps (1004; Alzet, Cupertino, CA, USA) were placed subcutaneously between the shoulder blades. Human PTH (1-34) $(40 \mu \mathrm{g} / \mathrm{kg}$ body weight per day) was infused with pumps for the indicated period $^{63}$.

Isolation of bone cells from adult bones. We isolated bone cells from female adult mice in accordance with a previously reported method ${ }^{22,64}$. Calvaria, humerus, ulnas/radius, hips, tibia, and femur bones were crushed, then washed with Hank's balanced salt solution. Bone tips were incubated in a $3 \mathrm{mg} / \mathrm{mL}$ solution of type I collagenase (Worthington) at $37^{\circ} \mathrm{C}$ for $25 \mathrm{~min}$. This incubation in collagenase solution was repeated twice (digestions 1-3). Next, bone pieces were incubated in $5 \mathrm{mM}$ ethylenediaminetetraacetic acid (EDTA) solution (digestion 4). Digestions 1-4 were subjected to flow cytometry for isolation of osteoblasts and MSCs.

Flow cytometry and cell sorting. Measurements were performed on a FACS Aria II (BD Biosciences, San Jose, CA, USA) and SH800 cell sorter (Sony, Tokyo, Japan), then analyzed using FlowJo software (TreeStar, Ashland, OR, USA). Single-cell suspensions were blocked with anti-CD16/32 antibody (BD Biosciences) for 10 min, followed by staining using flow cytometry (FACS) buffer (1× PBS, $4 \%$ heatinactivated FCS, and $2 \mathrm{mM}$ EDTA) for $15 \mathrm{~min}$. The antibodies for flow cytometry were as follows: anti-CD45-APC (1:50, 103112, BioLegend, San Diego, CA, USA), anti-CD45-FITC (1:50, 103108, BioLegend), anti-CD31-FITC (1:50, 102405, BioLegend), anti-F4/80-PE/Cy7 (1:50, 123113, BioLegend), anti-Ly6C-APC (1:50, 560595, BD Biosciences), anti-Ly6G-BV421 (1:50, 127627, BioLegend), anti-Sca1APC (1:50, 108111, BioLegend), anti-CD51-PE (1:100, 104105, BioLegend), Streptavidin-PE (1:100, 405203, BioLegend), and Streptavidin-FITC (1:50, 405201, BioLegend), Annexin V-FITC (1:50, 640905, BioLegend), and 7AAD (559525, BD Pharmingen, San Diego, CA, USA).

RNA-Seq analysis. For in vivo osteoblast analysis, $7 \mathrm{AAD}^{-} \mathrm{Lin}^{-} \mathrm{CD}^{-} 5^{-} \mathrm{ECFP}^{+}$cells from Col2.3-ECFP mice, treated with intermittent vehicle or PTH (1-34), were isolated using the BD FACSAria II instrument. Osteoblasts were harvested at $24 \mathrm{~h}$ after the final injection of PTH or vehicle. Total RNA was purified using an miRNeasy Micro Kit (Qiagen, Hilden, Germany). For in vitro MC3T3-E1 cell analysis, subconfluent cells were digested in QIAZOL (Qiagen) to extract total RNA, in accordance with the manufacturer's instructions. cDNA was generated using a SMART-Seq v. 4 Ultra Low Input RNA Kit (TaKaRa Clontech, Mountain View, CA, USA). Each cDNA sample was sheared (200-500 bp) using the Covaris S220 system (Covaris, Woburn, MA, USA) and prepared using KAPA Library Preparation Kits (Kapa Biosystems, Wilmington, MA, USA) in accordance with the manufacturer's instructions. Sequencing was performed on an Illumina HiSeq 2500 platform (Illumina, San Diego, CA, USA) in 75-base single-end mode; Illumina Casava v. 1.8.2 software was used for base-calling. Sequenced reads were mapped to the mouse reference genome sequences $(\mathrm{mm} 10)$ using TopHat v. 2.0.13 in combination with Bowtie2 v. 2.2.3 and SAMtools v. 0.1.19. The fragments per kilobase of transcript per million mapped reads (FPKM) were calculated using Cuffnorm v. 2.2.1. Bioinformatics analyses were performed using Integrated Differential Expression and Pathway Analysis (iDEP) v. 0.81, and Ingenuity Pathway Analysis software (Ingenuity Systems; Qiagen). The raw data have been deposited in the NCBI Gene Expression Omnibus database (GEO GSE145462).

Quantitative reverse transcription-polymerase chain reaction. Total RNA and cDNA were prepared using a Maxwell Simply RNA kit (Promega, Madison, WI, USA) and Superscript III reverse transcriptase (Invitrogen, Carlsbad, CA, USA). Real-time PCR was performed with a Thermal Cycler Dice Real-Time System (TaKaRa Bio, Shiga, Japan) using SYBR Premix EX Taq (TaKaRa Bio). The relative levels of the mRNAs of interest were calculated using the $2^{-\Delta \Delta C t}$ method. The mRNA level of Gapdh was used as the internal control. The primer sequences are listed in Supplementary Table S2.

Microstructure analysis. Micro-computed tomography (CT) was performed on the secondary trabeculae of distal femoral metaphyses. A cone-beam X-ray $\mu \mathrm{CT}$ system (ScanXmate-RB-0-SS150; Comscantecno, Yokohama, Japan) was used to obtain CT images of femoral bone samples. The settings were as follows: tube 
voltage, $70 \mathrm{kV}$; tube current, $0.1 \mathrm{~mA}$; and voxel size, $12.0 \mu \mathrm{m}$. The 3D images were reconstructed and analyzed using TRI/3D-BON software (Ratoc System Engineering, Kyoto, Japan). Regions of interest were drawn $500 \mu \mathrm{m}$ from the end of each epiphyseal growth plate to points $1.0 \mathrm{~mm}$ along the cortical wall. Bone was segmented from surrounding tissue using a threshold of $700 \mathrm{mgHA} / \mathrm{cm}^{3}$.

Bone histomorphometric analysis. Female mice were injected subcutaneously with $20 \mathrm{mg} / \mathrm{kg}$ body weight calcein (Sigma-Aldrich, St. Louis, MO, USA) at 72 and $24 \mathrm{~h}$ before sacrifice ${ }^{65}$. Tibias were collected and fixed in $70 \%$ ethanol. For histomorphometric analysis, tibias were embedded in glycolmethacrylate and toluidine blue staining was performed on sagittal sections. Calcein labeling was visualized using an inverted light microscope (DM-IL-LED-3; Leica Microsystems, Wetzlar, Germany). Quantitative bone histomorphometric measurements were performed in the secondary spongiosa using a Histometry-RT light microscope (System Supply, Nagano, Japan).

Measurement of bone metabolic markers. Female mice were fasted for $24 \mathrm{~h}$ before blood sampling. Blood was drawn via the inferior vena cava at $24 \mathrm{~h}$ after the final injection of PTH or vehicle. Blood specimens were centrifuged at $2000 \times g$ for $15 \mathrm{~min}$ in gel-separation tubes (TS-701; Sato Kasei Kogyosho Co. Ltd., Tochigi, Japan) to obtain serum. An enzyme-linked immunosorbent assay (ELISA) was used for quantitation of P1NP (Immunodiagnostics Systems, Fountain Hills, AZ, USA) and CTX (RatLaps TM; Immunodiagnostics Systems) in serum.

Cell culture of osteoblasts. MC3T3-E1 cells were purchased from the American Type Culture Collection (ATCC, Manassas, VA, USA). Primary osteoblasts were isolated from 2-day-old neonatal mouse calvaria as described previously ${ }^{66}$. Briefly, calvaria were subjected to five sequential digestions in $0.25 \%$ trypsin, $0.1 \% \mathrm{EDTA}$, and $0.32 \%$ collagenase type II (Worthington Biochemical Corp., Freehold, NJ, USA) solution for $25 \mathrm{~min}$ at $37^{\circ} \mathrm{C}$. Cell fractions $2-5$ were collected as primary osteoblasts. Adult primary osteoblasts derived from bone marrow were prepared as described previously ${ }^{67}$. Briefly, bone marrow cells from mouse long bones were seeded $\left(2.5 \times 10^{5}\right.$ cells per $\left.\mathrm{cm}^{2}\right)$ and expanded to obtain bone marrow stromal cells (BMSCs). Before differentiation, BMSCs were purified by negative selection with MACS CD45 microbeads (Miltenyi Biotec., Bergisch Gladbach, Germany).

For in vitro differentiation, osteoblasts were cultured with $50 \mu \mathrm{g} / \mathrm{mL}$ ascorbic acid (Wako Pure Chemical Co, Tokyo, Japan) and $10 \mathrm{mM} \beta$-glycerophosphate (Sigma-Aldrich) in $\alpha$-minimum essential medium (MEM) supplemented with $10 \%$ (v/v) fetal bovine serum, $0.22 \%$ sodium bicarbonate, $100 \mathrm{U} / \mathrm{mL}$ penicillin, and $100 \mu \mathrm{g} / \mathrm{mL}$ streptomycin.

ALP staining was performed using naphthol-ASMX as the substrate and Fast Blue RR salt as the coupler. ALP activity was detected using pNPP (4-nitrophenyl phosphate) with a TRACP \& ALP Assay Kit (TaKaRa) as a colorimetric substrate, then measured by spectrophotometry at $405 \mathrm{~nm}$. Staining and quantification of ALP activity were performed at 3 and 7 days after differentiation of MC3T3-E1 cells and primary osteoblasts, respectively.

Alizarin Red S staining and its quantification were performed using a Mineralization Quantification Kit (PG-Research, Tokyo, Japan). Staining was performed at 14 days after differentiation of MC3T3-E1 osteoblasts and primary osteoblasts. Quantification of Alizarin Red S staining was via extraction with formic acid. The amount of dye released was quantified by spectrophotometry at $405 \mathrm{~nm}$.

For the generation of conditioned medium, supernatants from subconfluent cultures of MC3T3-E1 cells were collected and passed through a $0.45 \mu \mathrm{m}$ filter to eliminate cells, diluted with one volume of $\alpha-M E M$, and used as conditioned medium.

For analysis of PTH1R signaling, osteoblasts were cultured under serum-free conditions for $12 \mathrm{~h}$ and topically treated with the following stimulators or inhibitors at the indicated concentrations:

Human PTH (1-34): PBS; $24.3 \mathrm{nM}$

Forskolin (Sigma-Aldrich): DMSO; $5 \mu \mathrm{M}$

PMA (Sigma-Aldrich): DMSO; $100 \mathrm{nM}$

A23187 (Sigma-Aldrich): DMSO; $5 \mu \mathrm{M}$

Rp-cAMPS (Sigma-Aldrich): DMSO; $25 \mu \mathrm{M}$

H89 (Calbiochem; Sigma-Aldrich): DMSO; $1 \mu \mathrm{M}$

U0126 (Cayman Chemical, Ann Arbor, MI, USA): DMSO; $10 \mu \mathrm{M}$

Retroviral and lentiviral gene transfer. The retroviral vector, pMX-Slpi-IRESPuro, was constructed by inserting EcoRI and XhoI fragments of the full-length mouse Slpi cDNA into pMX-IRES-Puro. Retroviral packaging was performed by transfecting the plasmids into Plat-E (Cell Biolabs, Inc., CA, USA) cells using FuGENE6 (Promega) ${ }^{6}$. Two days after inoculation, MC3T3-E1 cells or primary osteoblasts were incubated with viral supernatant from pMX-IRES-Puro and pMXSlpi-IRES-Puro virus-producing Plat-E cells, together with polybrene $(8 \mu \mathrm{g} / \mathrm{mL})$ for $12 \mathrm{~h}$. Seven days after infection, the cells were treated with puromycin $(10 \mu \mathrm{g} / \mathrm{mL})$ to select stable transformants.

To generate the MC3T3-E1-EGFP cell line, enhanced green fluorescent protein (EGFP) (pEGFP-C1; Clontech) was inserted into the CSII-EF-MCS vector (provided by Dr. Miyoshi of RIKEN-BRC, Tsukuba, Japan) and transfected into HEK293T cells (ATCC) with packaging plasmids ${ }^{69}$. Two days after inoculation,
MC3T3-E1 cells were incubated with lentiviral supernatant from virus-producing HEK293T cells, together with polybrene $(8 \mu \mathrm{g} / \mathrm{mL})$, for $12 \mathrm{~h}$. Stable transformants were selected using an SH800 cell sorter (Sony).

Evaluation of osteoblast proliferation. The proliferation of osteoblasts was assessed by counting cultured osteoblasts using a TC10 Automated Cell Counter (Bio-Rad, Hercules, CA, USA). Briefly, osteoblasts were seeded into six-well plates at $1 \times 10^{4} / \mathrm{cm}^{2}$. After 3 and 5 days, cells were suspended by incubation with $0.25 \%$ trypsin-EDTA (Sigma-Aldrich), and live cells were enumerated by trypan blue staining (Sigma-Aldrich).

Culture of primary osteoclasts. Nonadherent bone marrow cells derived from mice were seeded $(1.5-2.0 \times$ in a $10 \mathrm{~cm}$ Petri dish) and cultured in the presence of $30 \mathrm{ng} / \mathrm{mL}$ M-CSF (R\&D Systems, Minneapolis, MN, USA). After 3 days, adherent cells were collected in enzyme-free cell dissociation buffer (Millipore, Burlington, MA. USA) at $37^{\circ} \mathrm{C}$ and used as bone marrow macrophages (BMMs). BMMs were further cultured in the presence of $50 \mathrm{ng} / \mathrm{mL}$ soluble RANKL (Oriental Yeast Co., Ld., Tokyo, Japan) and $10 \mathrm{ng} / \mathrm{mL}$ M-CSF to generate osteoclasts ${ }^{70}$. Three days later, TRAP staining was conducted using an Acid Phosphatase, Leukocyte (TRAP) Kit (Sigma-Aldrich). $\mathrm{TRAP}^{+}$multinucleated $(>3$ nuclei) cells were regarded as mature osteoclasts.

To evaluate bone resorptive activity, osteoclasts were cultured on RepCell dishes for 5 days. Next, the cells were transferred to an Osteo Assay Surface (Corning, Corning, NY, USA). After $48 \mathrm{~h}$, resorption pits were observed under an inverted light microscope (DM-IL-LED-3; Leica Microsystems), and the binarized areas of resorption pits were measured using NIS Elements integrated software (Nikon, Tokyo, Japan).

Mass spectrometry. Protein samples in buffer solution were precipitated by methanol-chloroform addition and then dissolved in RapiGest (Waters, Milford, MA, USA). The samples were reduced with $10 \mathrm{mM}$ dithiothreitol followed by alkylation with iodoacetamide, then digested by trypsin and purified using a C18 tip (GL Science, Tokyo, Japan). Trypsin-digested peptides were subjected to liquid chromatography-tandem mass spectrometry, using an Aurora column (Ionoptics) on a Nano Elute nanoLC system coupled to a trapped ion mobility spectrometrytime-of-flight mass spectrometer (Bruker, Billerica, MA, USA). The column temperature was set to $50.0^{\circ} \mathrm{C}$. The mobile phase consisted of water with $0.1 \%$ formic acid (solvent $\mathrm{A}$ ) and acetonitrile containing $0.1 \%$ formic acid (solvent $\mathrm{B}$ ). Peptides were eluted at a gradient of $4-30 \%$ B for 20 min with a flow rate of $400 \mathrm{~nL} / \mathrm{min}$. The mass-scanning range was set from 100 to $1700 \mathrm{~m} / \mathrm{z}$ and a custom ion mobility revolution mode was used (range: $0.6-1.50 \mathrm{Vs} / \mathrm{cm}^{2}$ ). The ion spray voltage was set at $1.6 \mathrm{kV}$ in the positive-ion mode. The MS/MS spectra were acquired by automatic switching between MS and MS/MS modes. Data Analysis software (Bruker) was used to process the mass data. Peptides were identified by database searching using our in-house MASCOT Server (Matrix Science, London, UK). The precursor and fragment-searching mass tolerances were set to $20 \mathrm{ppm}$ and $0.05 \mathrm{Da}$, respectively. Carbamidomethylation of cysteine was set as a static modification, while the oxidation of methionine, acetylation of protein $\mathrm{N}$-term, and deamination of N,Q were set as variable modifications. The MASCOT Server search results were analyzed by Scaffold (Proteome Software Inc., Portland, OR, USA) and the results were limited to those with a GO annotation of "osteoblast." Finally, the quantitative values (normalized total spectra) of mock and immunoprecipitation samples were compared.

Immunoblotting analysis. Cells were lysed in RIPA buffer supplemented with protease inhibitor cocktail (Sigma-Aldrich). Conditioned media were mixed with four volumes of chilled acetone. Proteins were precipitated overnight at $-30^{\circ} \mathrm{C}$ and dissolved in RIPA buffer. Protein concentrations were measured by bicinchoninic acid protein assay (Thermo Scientific Inc., Waltham, MA, USA). Cell lysates were boiled in sodium dodecyl sulfate loading dye. Equal amounts of total protein were separated on $4-20 \%$ mini-Protean TGX gradient gels (Bio-Rad) and transferred to polyvinylidene difluoride membranes (Immobilon P). The membranes were incubated with the primary and secondary antibodies. Finally, bands were visualized with the electrochemiluminescence reagents (GE Healthcare, Chicago, IL, USA) and using an ImageQuant LAS- 4000 mini system (GE Healthcare). The antibodies for immunoblotting were as follows: anti- $\beta$-catenin $(1: 1000,610153, \mathrm{BD}$ Biosciences), anti-SLPI (1:1000, AF1735, R\&D Systems), anti-ROCK1 (1:1000, ab134181, Abcam, Cambridge, UK), anti-ROCK2 (1:1000, ab125025, Abcam) and anti- $\beta$-actin-HRP (1:5000, ab20272, Abcam), goat anti-mouse IgG-HRP (1:1000, sc-2005, Santa Cruz Biotechnology, Santa Cruz, CA, USA), rabbit anti-goat IgGHRP (1:1000, sc-2768, Santa Cruz Biotechnology).

Coculture of osteoblasts and osteoclasts. For time-lapse imaging, pMX-IRESPuro or pMX-Slpi-IRES-Puro were introduced into MC3T3-E1-EGFP cells. Stable transformants were selected with puromycin $(10 \mu \mathrm{g} / \mathrm{mL})$ for 3 days. Subsequently, the transformants were cocultured with primary osteoclasts. MC3T3-E1-EGFP cells and TRAP-tdTomato ${ }^{+}$osteoclasts were cocultured on a collagen gel-coated glass-bottom dish. MC3T3-E1 cells were cultured at a density of $750 \mathrm{cells} / \mathrm{cm}^{2}$, while mature osteoclasts were cultured at a 1:3 ratio of MC3T3-E1 cells to mature 
osteoclasts. To assess the effect of SLPI antibody on cell-cell contact, SLPI antibodies (AF1274, R\&D Systems; S0635, US Biological Life Sciences, Salem, MA, USA) $12.5 \mu \mathrm{g} / \mathrm{mL}$, or control goat IgG (Santa Cruz Biotechnology) were added before time-lapse imaging. After 3 days of coculture, time-lapse images were acquired using an A1 confocal microscope (Nikon). To assess the effect of Y27632 on cell-cell contact, MC3T3-E1 cells were exposed to $10 \mu \mathrm{M}$ Y27632 for $12 \mathrm{~h}$. The reagent was removed before coculture to prevent it from acting on osteoclasts. For tracking of cell-cell contact, images were first automatically binarized by Otsu's thresholding method using NIS Elements integrated software (Nikon). The surface tool of Imaris software (Bitplane, Zürich, Switzerland) was used to track cell movement. Only EGFP-stained surface objects $\geq 100-\mu \mathrm{m}^{2}$ and tdTomato-stained surface objects $\geq 1000-\mu \mathrm{m}^{2}$ were analyzed, to exclude extracellular vesicles from osteoblasts and tdTomato ${ }^{+}$mononuclear cells. A new channel representing the colocalized area of EGFP and tdTomato voxels was created automatically by the surface tool. Finally, the contact duration was determined by tracking this new channel using Imaris software.

To assess the effects of cell-cell interaction on the differentiation and activity of each cell type, osteoblasts (or MC3T3-E1 cells) and primary osteoclasts were first cultured separately. For osteogenic differentiation, primary osteoblasts and primary osteoclasts were each seeded at a density of $1.5 \times 10^{4} \mathrm{cells} / \mathrm{cm}^{2}$. Osteoblasts were differentiated for 14 days in the presence of RANKL and M-CSF. Osteoclasts were added at 3-day intervals during osteoblast differentiation. For osteoclast differentiation, osteoblasts (or MC3T3-E1 cells) and BMMs were seeded at densities of $1.5 \times 10^{4} / \mathrm{cm}^{2}$, and $3.75 \times 10^{4} / \mathrm{cm}^{2}$ respectively. Then, osteoclast differentiation was induced in the presence of RANKL and M-CSF for 5 days as described above. For the resorption pit assay, BMMs were differentiated into mature osteoclasts on $6 \mathrm{~cm}^{2}$ RepCell dishes for 5 days. Then osteoclasts were cocultured with MC3T3-E1 cells on an Osteo Assay Surface (Corning) for $48 \mathrm{~h}$ in the presence of RANKL and M-CSF to evaluate resorption activity.

To evaluate gene expression in coculture, MC3T3-E1-EGFP cells were differentiated for 14 days. BMMs derived from TRAP-tdTomato ${ }^{+}$mice were differentiated into mature osteoclasts on $6-\mathrm{cm}^{2}$ RepCell dishes for 5 days. For juxtacrine interactions, MC3T3-E1 cells and osteoclasts were mixed at a ratio of 2:1 and cocultured for $24 \mathrm{~h}$ at a density of $3.75 \times 10^{4} \mathrm{cells} / \mathrm{cm}^{2}$. For paracrine interactions, osteoclasts were cocultured for $24 \mathrm{~h}$, using a Millicell Hanging Cell culture insert with a 1- $\mu \mathrm{m}$ pore PET membrane (Millipore), with MC3T3-E1 cells at a density of $2.5 \times 10^{4} \mathrm{cell} / \mathrm{s} / \mathrm{cm}^{2}$. Inserts lacking cells were used as controls. Cells were collected using collagenase type II, and the $\mathrm{EGFP}^{+}$tdTomato- $^{-}$MC3T3E1 cells) and EGFP-tdTomato ${ }^{+}$(primary osteoclasts) fractions were sorted using an SH800 cell sorter (Sony).

Intravital multiphoton bone imaging. Calvarial bone tissues of female Col2.3ECFP/TRAP-tdTomato/Slpi $i^{\mathrm{wt} / \mathrm{wt}}$ and Col2.3-ECFP/TRAP-tdTomato/Slpi ${ }^{\mathrm{KO} / \mathrm{KO}}$ mice (12-20 weeks of age) were used for intravital bone imaging ${ }^{2}$. In this experiment, parietal lamina externa and intervening spongy bone (diploe) were observed. For surgery and continuous intravital imaging, mice were anesthetized with isoflurane (Escain; 2.5\% vaporized in an 80:20 mixture of $\mathrm{O}_{2}$ and air). The frontal and parietal bones were exposed and immobilized in a custom-made stereotactic holder.

The imaging system consisted of an upright multiphoton microscope (A1R MP+; Nikon) with a $25 \times$ water-immersion objective (CFI75 Apo 25XC W 1300; Nikon). The system was driven by lasers (Chameleon Vision II Ti: Sapphire; Coherent, Inc, Santa Clara, CA, USA); the main laser was tuned to $860 \mathrm{~nm}$ to detect ECFP and second harmonic generation (SHG), and the sub-laser was tuned to $1040 \mathrm{~nm}$ to detect tdTomato. Using a Nikon upright microscope, multi-fluorescence images were acquired directly using four external non-descanned detectors equipped with dichroic and emission filters including an infrared-cut filter (DM685), three dichroic mirrors (DM458, DM506, and DM605), and three emission filters (417/60 for the SHG image, $480 / 40$ for ECFP, $583 / 22$ for tdTomato).

Cell mixture analysis. Cell mixtures were evaluated using the $\mathrm{CMI}^{2}$. To obtain the tiling images, snapshot image stacks of 16 continuous visual fields were collected across the sagittal suture at depths of 50-200 $\mu \mathrm{m}$ below the skull bone surface (5$\mu \mathrm{m}$ vertical steps) using $1.0 \times$ zoom and $512 \times 512 \mathrm{X}-\mathrm{Y}$ resolution. Maximumintensity projection (MIP) images of tdTomato ${ }^{+}$mature osteoclasts and ECFP ${ }^{+}$ mature osteoblasts were stitched to produce a tiling image. The threshold for binarization was determined automatically using Otsu's method for a tiling image with gamma correction. Next, the MIP images were divided to create 16 images; only images in which the ratio of red area to cyan area ranged between 0.5 and 2 were analyzed. CMI indicates the degree of mixing of two cell types in the range from 0 to 1 , and was calculated by hierarchical clustering. Euclidean distances were calculated between each pixel in the binarized cell area, and Ward's method was used for the hierarchical clustering algorithm. Each pixel was initially considered a cluster of its own. Small clusters were combined sequentially into larger clusters, until all pixels converged into one cluster regardless of the color of individual pixels. The impurity of the clusters was calculated using a Gini-like formula, and $\mathrm{CMI}$ was defined as the area under the curve. Cell mixture analysis was performed using R software (ver. 3.6.0; R Development Core Team, Vienna, Austria) and the EBImage package (ver. 4.20.0).
Three-dimensional colocalization analysis. The numbers of osteoblast-osteoclast contacts were determined using Imaris. A Sobel filter was used to detect cell edges, in all depth slices and channels $(3 \times 3$; four directions for images acquired by the Nikon upright microscope with a $1.0 \times$ zoom). We next adjusted the raw images to yield edge-enhanced images. The surface tool in Imaris was used to perform automatic cell-surface segmentation of each cyan-positive and tdTomato-positive cell evident on intravital time-lapse bone imaging. Cyan-stained surface objects $\leq 125 \mu \mathrm{m}^{3}$ in volume, and tdTomato-stained surface objects $\leq 1000 \mu \mathrm{m}^{3}$ in volume, were not included in the analysis, as these objects were unlikely to represent cells. The surface tool was then used to detect osteoblast-osteoclast contacts, and to automatically create a new channel (yellow) for colocalized cyan and tdTomato voxels.

Histological analysis. Mice were perfused with $4 \%$ paraformaldehyde (PFA) for fixation and dissected femoral bones were further fixed with 4\% PFA and $20 \%$ sucrose for $4 \mathrm{~h}$ at $4{ }^{\circ} \mathrm{C}$. Samples were embedded in Super Cryoembedding medium (Section-LAB Co. Ltd) and frozen in chilled hexane (Wako) using dry ice ${ }^{2}$. Frozen samples were cut into $10 \mu \mathrm{m}$ sections with a cryostat (Leica, CM3050). Images were acquired with an Al confocal microscope (Nikon). Specific parameters (excitation laser wavelength, dichroic mirrors, and emission filters, respectively) were used for detecting fluorescence of ECFP (457 nm, DM515, and BP482/35) and tdTomato (561 nm, DM640, and BP595/50) (Nikon).

Statistical analysis. Statistical analysis was performed using unpaired two-tailed $t$ tests for comparisons between two groups, and using analysis of variance (ANOVA) with the Dunnett's, Tukey's, and Šidák's post hoc test for comparisons among three or more groups. Welch's ANOVA followed by Bonferroni's multiple comparisons test was used when the data demonstrated unequal variance. Statistical significance was recorded when $p<0.05$. Biological replicates comprised samples from different mice. The results are representative of more than three independent experiments. The minimum possible numbers of samples were used Mice were excluded if they exhibited any abnormalities in size, weight, or apparent symptoms of disease, before performing the experiments; however, no such phenomena were observed.

Reporting summary. Further information on research design is available in the Nature Research Reporting Summary linked to this article.

\section{Data availability}

The datasets are available from the corresponding authors on reasonable request. Raw RNA-Seq data is available from the Gene Expression Omnibus (accession number and hyperlinks: GSE145462)

Received: 3 April 2020; Accepted: 12 March 2021; Published online: 09 April 2021

\section{References}

1. Hattner, R., Epker, B. N. \& Frost, H. M. Suggested sequential mode of control of changes in cell behaviour in adult bone remodelling. Nature 206, 489-490 (1965).

2. Furuya, M. et al. Direct cell-cell contact between mature osteoblasts and osteoclasts dynamically controls their functions in vivo. Nat. Commun. 9, 300 (2018).

3. Neer, R. M. et al. Effect of parathyroid hormone (1-34) on fractures and bone mineral density in postmenopausal women with osteoporosis. N. Engl. J. Med. 344, 1434-1441 (2001).

4. Nakamura, T. et al. Randomized teriparatide [human parathyroid hormone (PTH) 1-34] once-weekly efficacy research (TOWER) trial for examining the reduction in new vertebral fractures in subjects with primary osteoporosis and high fracture risk. J. Clin. Endocrinol. Metab. 97, 3097-3106 (2012).

5. Huang, J. C. et al. PTH differentially regulates expression of RANKL and OPG. J. Bone Miner. Res. 19, 235-244 (2004).

6. Fu, Q., Manolagas, S. C. \& O'Brien, C. A. Parathyroid hormone controls receptor activator of NF-kappaB ligand gene expression via a distant transcriptional enhancer. Mol. Cell Biol. 26, 6453-6468 (2006).

7. Demiralp, B., Chen, H. L., Koh, A. J., Keller, E. T. \& McCauley, L. K. Anabolic actions of parathyroid hormone during bone growth are dependent on c-fos. Endocrinology 143, 4038-4047 (2002).

8. Koh, A. J. et al. Cells of the osteoclast lineage as mediators of the anabolic actions of parathyroid hormone in bone. Endocrinology 146, 4584-4596 (2005).

9. Black, D. M. et al. The effects of parathyroid hormone and alendronate alone or in combination in postmenopausal osteoporosis. N. Engl. J. Med. 349, 1207-1215 (2003) 
10. Finkelstein, J. S. et al. The effects of parathyroid hormone, alendronate, or both in men with osteoporosis. N. Engl. J. Med. 349, 1216-1226 (2003).

11. Wein, M. N. \& Kronenberg, H. M. Regulation of bone remodeling by parathyroid hormone. Cold Spring Harb. Perspect. Med. 8 (2018).

12. Ashcroft, G. S. et al. Secretory leukocyte protease inhibitor mediates nonredundant functions necessary for normal wound healing. Nat. Med. 6 , $1147-1153$ (2000).

13. Taggart, C. C., Greene, C. M., McElvaney, N. G. \& O'Neill, S. J. Secretory leucoprotease inhibitor prevents lipopolysaccharide-induced IkappaBalpha degradation without affecting phosphorylation or ubiquitination. J. Biol. Chem. 277, 33648-33653 (2002).

14. Greene, C. M., McElvaney, N. G., O’Neill, S. J. \& Taggart, C. C. Secretory leucoprotease inhibitor impairs Toll-like receptor 2- and 4-mediated responses in monocytic cells. Infect. Immun. 72, 3684-3687 (2004).

15. Taggart, C. C. et al. Secretory leucoprotease inhibitor binds to NF-kappaB binding sites in monocytes and inhibits p65 binding. J. Exp. Med. 202, 1659-1668 (2005).

16. Zhu, J. et al. Conversion of proepithelin to epithelins: roles of SLPI and elastase in host defense and wound repair. Cell 111, 867-878 (2002).

17. Bi, Y. et al. Biglycan deficiency increases osteoclast differentiation and activity due to defective osteoblasts. Bone 38, 778-786 (2006).

18. Iida-Klein, A. et al. Anabolic action of parathyroid hormone is skeletal site specific at the tissue and cellular levels in mice. J. Bone Miner. Res. 17, 808-816 (2002).

19. Hurley, M. M. et al. Impaired bone anabolic response to parathyroid hormone in Fgf2 $2^{-1-}$ and Fgf2 ${ }^{+-}$mice. Biochem. Biophys. Res. Commun. 341, 989-994 (2006).

20. Tam, C. S., Heersche, J. N., Murray, T. M. \& Parsons, J. A. Parathyroid hormone stimulates the bone apposition rate independently of its resorptive action: differential effects of intermittent and continuous administration. Endocrinology 110, 506-512 (1982).

21. Seita, J. et al. Gene Expression Commons: an open platform for absolute gene expression profiling. PLoS One 7, e40321 (2012).

22. Schepers, K., Hsiao, E. C., Garg, T., Scott, M. J. \& Passegué, E. Activated Gs signaling in osteoblastic cells alters the hematopoietic stem cell niche in mice. Blood 120, 3425-3435 (2012).

23. Nakamura, A. et al. Increased susceptibility to LPS-induced endotoxin shock in secretory leukoprotease inhibitor (SLPI)-deficient mice. J. Exp. Med. 197, 669-674 (2003).

24. Abou-Samra, A. B. et al. Expression cloning of a common receptor for parathyroid hormone and parathyroid hormone-related peptide from rat osteoblast-like cells: a single receptor stimulates intracellular accumulation of both cAMP and inositol trisphosphates and increases intracellular free calcium. Proc. Natl Acad. Sci. USA. 89, 2732-2736 (1992).

25. Gesty-Palmer, D. et al. Distinct beta-arrestin- and G protein-dependent pathways for parathyroid hormone receptor-stimulated ERK1/2 activation. J. Biol. Chem. 281, 10856-10864 (2006).

26. Grütter, M. G., Fendrich, G., Huber, R. \& Bode, W. The $2.5 \AA$ X-ray crystal structure of the acid-stable proteinase inhibitor from human mucous secretions analysed in its complex with bovine alpha-chymotrypsin. EMBO J. 7, 345-351 (1988).

27. Komori, T. et al. Targeted disruption of Cbfal results in a complete lack of bone formation owing to maturational arrest of osteoblasts. Cell 89, 755-764 (1997).

28. Ducy, P., Zhang, R., Geoffroy, V., Ridall, A. L. \& Karsenty, G. Osf2/Cbfa1: a transcriptional activator of osteoblast differentiation. Cell 89, 747-754 (1997).

29. Lentsch, A. B. et al. Inhibition of NF-kappaB activation and augmentation of IkappaBbeta by secretory leukocyte protease inhibitor during lung inflammation. Am. J. Pathol. 154, 239-247 (1999).

30. Chang, J. et al. Inhibition of osteoblastic bone formation by nuclear factorkappaB. Nat. Med. 15, 682-689 (2009).

31. Noguchi, T. et al. Progranulin plays crucial roles in preserving bone mass by inhibiting TNF- $\alpha$-induced osteoclastogenesis and promoting osteoblastic differentiation in mice. Biochem. Biophys. Res. Commun. 465, 638-643 (2015).

32. Jilka, R. L. et al. Increased bone formation by prevention of osteoblast apoptosis with parathyroid hormone. J. Clin. Investig. 104, 439-446 (1999).

33. Iotsova, V. et al. Osteopetrosis in mice lacking NF-kappaB1 and NF-kappaB2. Nat. Med. 3, 1285-1289 (1997).

34. Everts, V., Beertsen, W. \& Schröder, R. Effects of the proteinase inhibitors leupeptin and E-64 on osteoclastic bone resorption. Calcif. Tissue Int. 43, 172-178 (1988).

35. Everts, V. et al. Degradation of collagen in the bone-resorbing compartment underlying the osteoclast involves both cysteine-proteinases and matrix metalloproteinases. J. Cell Physiol. 150, 221-231 (1992).

36. Brömme, D. \& Okamoto, K. Human cathepsin O2, a novel cysteine protease highly expressed in osteoclastomas and ovary molecular cloning, sequencing and tissue distribution. Biol. Chem. Hoppe Seyler 376, 379-384 (1995).
37. Jeong, S. J. et al. Secretory leukocyte protease inhibitor (SLPI) increases focal adhesion in MC3T3 osteoblast on titanium surface. J. Nanosci. Nanotechnol. 15, 200-204 (2015).

38. Subramaniyam, D. et al. Secretory leukocyte protease inhibitor inhibits neutrophil apoptosis. Respirology 16, 300-307 (2011).

39. $\mathrm{Yu}, \mathrm{X}$. et al. CCR1 chemokines promote the chemotactic recruitment, RANKL development, and motility of osteoclasts and are induced by inflammatory cytokines in osteoblasts. J. Bone Miner. Res. 19, 2065-2077 (2004).

40. Li, D. et al. Platelet-derived growth factor BB enhances osteoclast formation and osteoclast precursor cell chemotaxis. J. Bone Miner. Metab. 35, 355-365 (2017).

41. Negishi-Koga, T. et al. Suppression of bone formation by osteoclastic expression of semaphorin 4D. Nat. Med. 17, 1473-1480 (2011).

42. Roy, A. D. et al. Optogenetic activation of Plexin-B1 reveals contact repulsion between osteoclasts and osteoblasts. Nat. Commun. 8, 15831 (2017).

43. Yang, L. et al. Rho GTPase Cdc42 coordinates hematopoietic stem cell quiescence and niche interaction in the bone marrow. Proc. Natl Acad. Sci. USA 104, 5091-5096 (2007).

44. Hirai, F. et al. Small GTPase Rho signaling is involved in betal integrinmediated up-regulation of intercellular adhesion molecule 1 and receptor activator of nuclear factor kappaB ligand on osteoblasts and osteoclast maturation. Biochem. Biophys. Res. Commun. 356, 279-285 (2007).

45. Tanaka, Y. et al. Intercellular adhesion molecule 1 discriminates functionally different populations of human osteoblasts: characteristic involvement of cell cycle regulators. J. Bone Miner. Res. 15, 1912-1923 (2000)

46. Shi, C., Zhang, H., Louie, K., Mishina, Y. \& Sun, H. BMP signaling mediated by BMPR1A in osteoclasts negatively regulates osteoblast mineralization through suppression of Cx43. J. Cell Biochem. 118, 605-614 (2017).

47. Townes, P. L. \& Holfreter, J. Directed movements and selective adhesion of embryonic amphibian cells. J. Exp. Zool. 128, 53-120 (1955).

48. Sims, N. A. \& Vrahnas, C. Regulation of cortical and trabecular bone mass by communication between osteoblasts, osteocytes and osteoclasts. Arch. Biochem. Biophys. 561, 22-28 (2014).

49. Sims, G. P., Rowe, D. C., Rietdijk, S. T., Herbst, R. \& Coyle, A. J. HMGB1 and RAGE in inflammation and cancer. Annu. Rev. Immunol. 28, 367-388 (2010).

50. Bronckaers, A. et al. Identification of aspartic acid-203 in human thymidine phosphorylase as an important residue for both catalysis and non-competitive inhibition by the small molecule "crystallization chaperone" 5 -O-tritylinosine (KIN59). Biochem. Pharmacol. 78, 231-240 (2009).

51. Miyazono, K., Okabe, T., Urabe, A., Takaku, F. \& Heldin, C. H. Purification and properties of an endothelial cell growth factor from human platelets. $J$. Biol. Chem. 262, 4098-4103 (1987).

52. Hotchkiss, K. A., Ashton, A. W. \& Schwartz, E. L. Thymidine phosphorylase and 2-deoxyribose stimulate human endothelial cell migration by specific activation of the integrins alpha 5 beta 1 and alpha V beta 3. J. Biol. Chem. 278, 19272-19279 (2003).

53. Cho, S. W. et al. Osteal macrophages support physiologic skeletal remodeling and anabolic actions of parathyroid hormone in bone. Proc. Natl Acad. Sci. USA 111, 1545-1550 (2014)

54. Onan, D. et al. The chemokine Cxcl1 is a novel target gene of parathyroid hormone (PTH)/PTH-related protein in committed osteoblasts. Endocrinology 150, 2244-2253 (2009).

55. Hojilla, C. V., Mohammed, F. F. \& Khokha, R. Matrix metalloproteinases and their tissue inhibitors direct cell fate during cancer development. Br. J. Cancer 89, 1817-1821 (2003).

56. Phillips-Mason, P. J., Craig, S. E. \& Brady-Kalnay, S. M. A protease storm cleaves a cell-cell adhesion molecule in cancer: multiple proteases converge to regulate PTPmu in glioma cells. J. Cell Biochem. 115, 1609-1623 (2014).

57. Lutz, D. et al. Proteolytic cleavage of transmembrane cell adhesion molecule L1 by extracellular matrix molecule Reelin is important for mouse brain development. Sci. Rep. 7, 15268 (2017).

58. Wen, J. et al. Secretory leukocyte protease inhibitor (SLPI) expression and tumor invasion in oral squamous cell carcinoma. Am. J. Pathol. 178, 2866-2878 (2011)

59. Recker, R. R. et al. Issues in modern bone histomorphometry. Bone 49, 955-964 (2011)

60. Takyar, F. M. et al. EphrinB2/EphB4 inhibition in the osteoblast lineage modifies the anabolic response to parathyroid hormone. J. Bone Miner. Res. 28, 912-925 (2012).

61. Terauchi, M. et al. T lymphocytes amplify the anabolic activity of parathyroid hormone through Wnt10b signaling. Cell Metab. 10, 229-240 (2009).

62. Kikuta, J. et al. Dynamic visualization of RANKL and Th17-mediated osteoclast function. J. Clin. Investig. 123, 866-873 (2013).

63. Iida-Klein, A. et al. Short-term continuous infusion of human parathyroid hormone 1-34 fragment is catabolic with decreased trabecular connectivity density accompanied by hypercalcemia in C57BL/J6 mice. J. Endocrinol. 186, 549-557 (2005) 
64. Stern, A. R. et al. Isolation and culture of primary osteocytes from the long bones of skeletally mature and aged mice. Biotechniques 52, 361-373 (2012).

65. Maridas, D. E. et al. Progenitor recruitment and adipogenic lipolysis contribute to the anabolic actions of parathyroid hormone on the skeleton. FASEB J. 33, 2885-2898 (2019).

66. Bakker, A. D. \& Klein-Nulend, J. Osteoblast isolation from murine calvaria and long bones. Methods Mol. Biol. 816, 19-29 (2012).

67. Hayashi, M. et al. Osteoprotection by semaphorin 3A. Nature 485, 69-74 (2012).

68. Morita, S., Kojima, T. \& Kitamura, T. Plat-E: an efficient and stable system for transient packaging of retroviruses. Gene Ther. 7, 1063-1066 (2000).

69. Sakabe, M. et al. YAP/TAZ-CDC42 signaling regulates vascular tip cell migration. Proc. Natl Acad. Sci. USA. 114, 10918-10923 (2017).

70. Marino, S., Logan, J. G., Mellis, D. \& Capullid, M. Generation and culture of osteoclasts. Bonekey Rep. 3, 570 (2014).

\section{Acknowledgements}

This work was supported by funding from Innovative Drug Discovery and Development Project (Japan Agency for Medical Research and Development [AMED] under Grant Number JP20am0401009 to M.I. and J.K.), CREST, Japan Science and Technology Agency (to M.I.), Grants-in-Aid for Scientific Research (S) from the Japan Society for the Promotion of Science (JSPS to M.I.); PRIME (Japan Agency for Medical Research and Development [AMED] under Grant Number JP20gm6210005 to J.K.), a Grant-in-Aid for Young Scientists from JSPS (to J.K.), and grants from the Uehara Memorial Foundation (to M.I.), the Kanae Foundation for the Promotion of Medical Sciences (to M.I), the Mochida Memorial Foundation (to M.I.), and the Takeda Science Foundation (to M.I.). This research was supported by research grants from Asahi Kasei Pharma Corporation.

\section{Author contributions}

A.M. and M.I. conceived the study. A.M. and J.K designed the experiments. A.M. performed the imaging experiments and data analysis with assistance from J.K., K.N., T.S., M.U., T.H., K.H., H.T., and M.F., S.S., and H.M. contributed to the imaging data analysis. A.N. provided the Slpi-KO mouse line. D.O. performed the RNA-Seq analysis. F.S. and
A.N. performed the proteomics analysis. T.Y. and R.T.-K. discussed the experiments and results. A.M. wrote the initial draft. J.K. and M.I. revised the final draft.

\section{Competing interests}

T.Y. and R.T.-K. are full-time employees of Asahi Kasei Pharma Corporation. The other authors have no conflict of interest.

\section{Additional information}

Supplementary information The online version contains supplementary material available at https://doi.org/10.1038/s41467-021-22402-x.

Correspondence and requests for materials should be addressed to J.K. or M.I.

Peer review information Nature Communications thanks Koichi Matsuo and the other, anonymous, reviewer(s) for their contribution to the peer review of this work.

Reprints and permission information is available at http://www.nature.com/reprints

Publisher's note Springer Nature remains neutral with regard to jurisdictional claims in published maps and institutional affiliations.

Open Access This article is licensed under a Creative Commons Attribution 4.0 International License, which permits use, sharing, adaptation, distribution and reproduction in any medium or format, as long as you give appropriate credit to the original author(s) and the source, provide a link to the Creative Commons license, and indicate if changes were made. The images or other third party material in this article are included in the article's Creative Commons license, unless indicated otherwise in a credit line to the material. If material is not included in the article's Creative Commons license and your intended use is not permitted by statutory regulation or exceeds the permitted use, you will need to obtain permission directly from the copyright holder. To view a copy of this license, visit http://creativecommons.org/ licenses/by/4.0/

(C) The Author(s) 2021 